\title{
Minimum light numbers in the $\sigma$-game and lit-only $\sigma$-game on unicyclic and grid graphs
}

\author{
John Goldwasser* Xinmao Wang ${ }^{\dagger} \quad$ Yaokun $\mathrm{Wu}^{\ddagger}$
}

Submitted: Mar 2, 2011; Accepted: Oct 18, 2011; Published: Oct 31, 2011

Mathematics Subject Classification: 05C05; 05C57; 37B99; 90C27; 91A43

\begin{abstract}
Consider a graph each of whose vertices is either in the ON state or in the OFF state and call the resulting ordered bipartition into $\mathrm{ON}$ vertices and $\mathrm{OFF}$ vertices a configuration of the graph. A regular move at a vertex changes the states of the neighbors of that vertex and hence sends the current configuration to another one. A valid move is a regular move at an ON vertex. For any graph $G$, let $\mathcal{D}(G)$ be the minimum integer such that given any starting configuration $\mathrm{x}$ of $G$ there must exist a sequence of valid moves which takes $\mathbf{x}$ to a configuration with at most $\ell+\mathcal{D}(G)$ ON vertices provided there is a sequence of regular moves which brings $\mathbf{x}$ to a configuration in which there are $\ell$ ON vertices. The shadow graph $\mathcal{S}(G)$ of a graph $G$ is obtained from $G$ by deleting all loops. We prove that $\mathcal{D}(G) \leq 3$ if $\mathcal{S}(G)$ is unicyclic and give an example to show that the bound 3 is tight. We also prove that $\mathcal{D}(G) \leq 2$ if $G$ is a two-dimensional grid graph and $\mathcal{D}(G)=0$ if $\mathcal{S}(G)$ is a two-dimensional grid graph but not a path and $G \neq \mathcal{S}(G)$.
\end{abstract}

\section{Definitions and background}

A graph $G$ is a pair of sets consisting of its vertex set $V(G)$ and its edge set $E(G)$ such that $E(G) \subseteq\left(\begin{array}{c}V(G) \\ 2\end{array}\right) \cup V(G)$ and we say that there is a loop at a vertex $v$ of $G$ provided $\{v\} \in E(G)$. When $u \neq v$, we often denote an edge $\{u, v\}$ by $u v$; a singleton set $\{u\}$ is mostly just written as $u$. Two different vertices $u$ and $v$ of $G$ are adjacent provided $u v \in E(G)$ and $v$ is adjacent to itself if $v \in V(G) \cap E(G)$ (so there is a loop at $v$ ). The set of vertices adjacent to a given vertex $v$ in $G$ is designated by $N_{G}(v)$ and called the

*Department of Mathematics, West Virginia University, Morgantown, WV 26506, USA. Email: jgoldwas@math. wvu . edu.

${ }^{\dagger}$ Department of Mathematics, University of Science and Technology of China, Hefei, Anhui 230026, China. Email: xinmao@ustc.edu.cn.

${ }_{\ddagger}^{\ddagger}$ Department of Mathematics, Shanghai Jiao Tong University, Shanghai 200240, China. Email: ykwu@sjtu.edu.cn. Fax: 86-21-54743152. Corresponding author. 
set of neighbors of $v$ in $G$. For $S \subseteq V(G)$, we put $G[S]$ to be the graph with vertex set $S$ and edge set $E(G) \cap\left(\left(\begin{array}{l}S \\ 2\end{array}\right) \cup S\right)$. For any $v \in V(G)$, we use the abbreviation $G-v$ for $G[V(G) \backslash\{v\}]$. The degree of a vertex $v$ in a graph $G$ is defined to be the number of edges in $E(G) \backslash V(G)$ that contain $v$ and we will use the notation $\operatorname{deg}_{G}(v)$ for it. We say that $v$ is a branch vertex of $G$ if $\operatorname{deg}_{G}(v) \geq 3$.

For any $k$ positive integers $m_{1}, \ldots, m_{k}$, the $k$-dimensional grid graph $G_{m_{1}, \ldots, m_{k}}$ has vertex set $\left\{v_{i_{1}, \ldots, i_{k}}: 1 \leq i_{1} \leq m_{1}, \ldots, 1 \leq i_{k} \leq m_{k}\right\}$ and $\left\{v_{i_{1}, \ldots, i_{k}}, v_{j_{1}, \ldots, j_{k}}\right\} \in E\left(G_{m_{1}, \ldots, m_{k}}\right)$ if and only if $\sum_{t=1}^{k}\left(i_{t}-j_{t}\right)^{2}=1$. The graph $G_{n}$ is often called an $n$-path and denoted $\left[v_{1}, \ldots, v_{n}\right]$; see Fig. 1 . For any $n \geq 3$, the graph obtained from the path $\left[v_{1}, \ldots, v_{n}\right]$ by adding an edge $v_{1} v_{n}$ is referred to as an $n$-cycle and is denoted $\left\langle v_{1}, \ldots, v_{n}\right\rangle$. Note that a 4-cycle is nothing but $G_{2,2}$. A unicyclic graph is a loopless connected graph containing exactly one cycle.

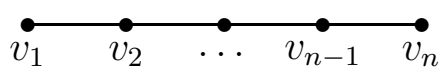

Figure 1: An $n$-path $\left[v_{1}, \ldots, v_{n}\right]$.

The shadow graph of a graph $G$, which we denote by $\mathcal{S}(G)$, is the (loopless) graph with vertex set $V(G)$ and edge set $E(G) \backslash V(G)$. If $\mathcal{S}(G)$ is a tree, we call $G$ a pseudo-tree. Similarly, we can talk about a pseudo-cycle and a pseudo-unicyclic graph, etc..

Let $\mathbb{F}_{2}$ be the binary field and we refer to any element $\mathbf{x}$ of $\mathbb{F}_{2}^{V(G)}$ as a configuration of $G$. We say that $v \in V(G)$ is ON in $\mathbf{x}$ if $\mathbf{x}(v)=1$ and is OFF in $\mathbf{x}$ if $\mathbf{x}(v)=0$ and hence we can also regard a configuration as an assignment of states ON (1) or OFF (0) to vertices of $G$, or simply an ordered bipartition of $V(G)$ into ON vertex set and OFF vertex set. The light number $L(\mathbf{x})$ of a configuration $\mathbf{x}$ is the number of $O N$ vertices in $\mathbf{x}$, namely $L(\mathbf{x})=|\operatorname{supp}(\mathbf{x})|$, where $\operatorname{supp}(\mathbf{x})$ means the support of the function $\mathbf{x}$. For any $\mathbf{x} \in \mathbb{F}_{2}^{V(G)}$ and any $U \subseteq V(G), \mathbf{x}_{U}$ is the restriction of $\mathbf{x}$ on $U$, namely the image of $\mathbf{x}$ under the natural projection from $\mathbb{F}_{2}^{V(G)}$ to $\mathbb{F}_{2}^{U}$. For any $S \subseteq V(G), \chi_{S} \in \mathbb{F}_{2}^{V(G)}$ is the characteristic vector of $S$. Note that $\chi_{S}+\chi_{Q}=\chi_{S \triangle Q}$ where $\triangle$ stands for taking the symmetric difference of two sets. We will write $\chi_{\{v\}}$ simply as $\chi_{v}$. It is clear that each configuration $\mathbf{x}$ is just the characteristic vector of $\operatorname{supp}(\mathbf{x})$. In a graphical representation of a configuration, we often use a circle for a vertex in the OFF state and a bullet for a vertex in the ON state; see Fig. 2 for an example.

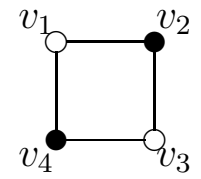

Figure 2: A cycle of length 4 with configuration $\chi_{\left\{v_{2}, v_{4}\right\}}$.

A regular move (regular toggling or regular switching) at a vertex $v$ on a graph $G$ transforms a configuration $\mathbf{x}$ to $\mathbf{x}+\chi_{N_{G}(v)}$ and we write $\mathbf{x} \rightarrow_{G} \mathbf{y}$ to designate that we 
can make successive moves to go from $\mathbf{x}$ to $\mathbf{y}$. In the $\sigma$-game on $G$ [42], we are given a configuration $\mathbf{x}$ and aim to find a $\mathbf{y}$ such that $\mathbf{x} \rightarrow_{G} \mathbf{y}$ and that $L(\mathbf{y})$ is as small as possible. In the literature, if there is a loop at each vertex of $G$, the operation is sometimes called closed neighborhood switching and the game is generally called the $\sigma^{+}$-game. The phase space (game digraph) $\mathbb{P S}(G)$ of the $\sigma$-game on a graph $G$ is the arc-labeled symmetric digraph whose vertex set (phases) is the set of configurations on $G$ and whose phase transitions (labeled-arcs) consist of a vertex $v$ (the label) and corresponding regular move at $v$ from a configuration to a different configuration (the arc).

A lit-only move (lit-only toggling) at a vertex $v$ on a graph $G$ transforms a configuration $\mathbf{x}$ to $\mathbf{x}+\mathbf{x}(v) \chi_{N_{G}(v)}$. Clearly, the lit-only toggling at $v$ will not change the configuration if $v$ is $\mathrm{OFF}$ and will change the states of all neighbors of $v$ if $v$ is $\mathrm{ON}$. We say that a lit-only move at $v$ applied on a configuration $\mathbf{x}$ is valid if $\mathbf{x}(v) \chi_{N_{G}(v)}$ is nonzero. For brevity, a valid move will often be referred to as a move/pushing/toggling in the sequel. Note that the set of valid moves forms a special subclass of regular moves. The set of valid moves gives rise to an asynchronous discrete dynamical system, called the lit-only $\sigma$-game on $G$, and the dynamical behavior of this system is captured by its phase space (game digraph) $\mathbb{P S}^{*}(G)$, the arc-labeled digraph whose vertex set (phases) is the set of configurations on $G$ and whose phase transitions (labeled-arcs) consist of a vertex $v$ (the label) and corresponding valid move at $v$ from a configuration to a different configuration (the arc). See Appendix A for a description of $\mathbb{P S}^{*}(G)$ where $G$ runs through all 4-cycles with some loops attached. Note that $\mathbb{P S}^{*}(G)$ is a subdigraph of $\mathbb{P S}(G)$. For any two configurations $\mathbf{x}$ and $\mathbf{y}$ of $G$, we write $\mathbf{x} \stackrel{*}{\rightarrow}_{G} \mathbf{y}$ to mean that we can make successive lit-only moves to go from $\mathbf{x}$ to $\mathbf{y}$, namely, there exists a walk from $\mathbf{x}$ to $\mathbf{y}$ in $\mathbb{P S}^{*}(G)$. Moreover, if there is a walk from $\mathbf{x}$ to $\mathbf{y}$ in $\mathbb{P S}^{*}(G)$ on which a label $v$ is read an odd number of times if and only if $v \in S \subseteq V(G)$, we will record this by $\mathbf{x}_{S}^{*} \underset{G}{*} \mathbf{y}$.

The lit-only $\sigma$-game and its closely related variants have been studied not only for fun by amateurs [40] but are also studied by mathematicians for mathematical fun $[8,10,25$, $26,44,45,46,48]$ and from the perspectives of error-correcting codes and combinatorial game theory $[12,13,14,15,17]$, Lie algebras and Coxeter groups $[4,5,6,7,29,30,31$, $32,39]$, statistical physics of social balance [33, 34], and general reachability analysis [27]. The study of the $\sigma$-game has a longer history than that of the lit-only $\sigma$-game and is still mushrooming; see $[1,2,3,9,10,11,16,18,19,20,21,22,23,24,25,28,35,36,40,41$, 42, 43, 47, 49] and their references.

For any $\mathbf{x} \in \mathbb{F}_{2}^{V(G)}$, we define its minimum light number for the $\sigma$-game on $G$ to be

$$
M L_{G}(\mathbf{x})=\min _{\mathbf{x} \rightarrow G \mathbf{y}} L(\mathbf{y})
$$

and define its minimum light number for the lit-only $\sigma$-game on $G$, also called its lit-only minimum light number, to be

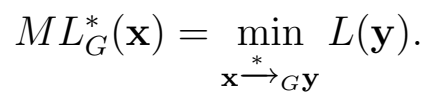

The minimum light number for the $\sigma$-game on $G$, denoted $M L(G)$, is the worst result a 
smart player can encounter, namely

$$
M L(G)=\max _{\mathbf{x} \in \mathbb{F}_{2}^{V(G)}} M L_{G}(\mathbf{x}) .
$$

We let $M L^{*}(G)$ denote the minimum light number for the lit-only $\sigma$-game on $G$, that is,

$$
M L^{*}(G)=\max _{\mathbf{x} \in \mathbb{F}_{2}^{V(G)}} M L_{G}^{*}(\mathbf{x})
$$

It is trivial to see that $M L_{G}(\mathbf{x}) \leq M L_{G}^{*}(\mathbf{x})$ and $M L(G) \leq M L^{*}(G)$. A graph $G$ is nonsingular (singular) provided its adjacency matrix is nonsingular (singular) over $\mathbb{F}_{2}$, namely provided $M L(G)=0(M L(G)>0)$.

Lemma 1. [45, Theorem 12] If $G$ is obtained from the $n$-path $\left[v_{1}, v_{2}, \ldots, v_{n}\right]$ by adding zero or more loops, then any configuration $\mathbf{x}$ of $G$ can be transformed to a configuration with light number at most one by a series of valid moves inside $\left\{v_{1}, v_{2}, \ldots, v_{n-1}\right\}$.

Example 2. Suppose $G$ is the n-path. Then,

$$
M L^{*}(G)=1, \quad M L(G)= \begin{cases}1, & \text { if } n \text { is odd } \\ 0, & \text { if } n \text { is even } .\end{cases}
$$

On the other hand, if $G$ is the $n$-cycle then

$$
M L^{*}(G)=2 \text { and } M L(G)= \begin{cases}1, & \text { if } n \text { is odd } \\ 2, & \text { if } n \text { is even }\end{cases}
$$

It turns out that many algebraic/combinatorial tools are useful to estimate $M L_{G}(\mathbf{x})$ for any/some $\mathbf{x} \in \mathbb{F}_{2}^{V(G)}$ and hence also $M L(G)$. The estimation of $M L_{G}^{*}(\mathbf{x})$ and $M L^{*}(G)$ is a relatively new task and seems that not many tools apply well here. It thus becomes natural to study the parameter

$$
\mathcal{D}(G)=\max _{\mathbf{x} \in \mathbb{F}_{2}^{V(G)}}\left(M L_{G}^{*}(\mathbf{x})-M L_{G}(\mathbf{x})\right)
$$

as the massive literature on the $\sigma$-game may already provide us good information on $M L_{G}(\mathbf{x})$. If the graph $G$ is nonsingular, we have $M L(G)=0$ and so $\mathcal{D}(G)=M L^{*}(G)$. More generally, it is not hard to verify the next observation.

Theorem 3. [45, Theorem 2] For any graph $G$, the inequality $M L^{*}(G) \leq M L(G)+\mathcal{D}(G)$ holds. That is, $\mathcal{D}(G)$ is an upper bound of $M L^{*}(G)-M L(G)$.

For any integer $m>1$, a complete m-partite graph is a loopless graph whose vertex set can be partitioned into $m$ nonempty parts such that two vertices are adjacent if and only if they belong to different parts. 
Example 4. Let $k$ and $t$ be positive integers with $m=2 k+1$ and $n=m t$, and let $G$ be the complete equi-m-partite graph with $n$ vertices ( $t$ vertices in each part). If $\mathbf{x}$ is the configuration with all vertices $O N$ in $k+1$ parts and all OFF in the other $k$ parts, then $M L^{*}(\mathbf{x})=\frac{k+1}{2 k+1} n$, while $M L(\mathbf{x})=0$ if $k$ is odd. Hence $\mathcal{D}(G)$ can be as large as $2 n / 3$ when $k=1$.

We have found that $M L_{G}^{*}(\mathbf{x}) \leq \frac{2|V(G)|}{3}$ for any configuration $\mathbf{x}$ of any graph $G$ and the equality holds only when each connected component of $G$ is a complete equi-tripartite graph and when two partite sets of each such component are $\mathrm{ON}$ in $\mathbf{x}$ and the remaining one partite set of each such component is OFF. This result along with Example 4 and some further research suggest the following conjecture. The reader may like to compare it with [45, Conjecture 4].

Conjecture 5. Let $G$ be a connected graph on $n$ vertices. If $\mathcal{D}(G)>n / 2$ then $G$ is a complete $m$-partite graph for some positive integer $m \equiv 3(\bmod 4)$.

In spite of the possible large values of $\mathcal{D}(G)$ for certain graphs $G$, the existence of certain local structures guarantees that $\mathcal{D}(G)$ will be small in some classes of graphs. This paper is a continuation of the work in [45] towards further understanding this observed small difference on many graphs. Almost all earlier work focuses on either graphs where there are no loops (usually called the regular and lit-only $\sigma$-games) or where every vertex has a loop (usually called the regular and lit-only $\sigma^{+}$-games). When there are no loops, both the regular and lit-only games can be viewed as permutation groups acting on the set of configurations on a graph. In the regular $\sigma$-game the group is abelian, so there are induced orbits all of the same size. In the lit-only $\sigma$-game, the group is a nonabelian subgroup of $G L\left(n, \mathbb{F}_{2}\right)$ with orbits of non-uniform size [25]. For the regular $\sigma^{+}$-game, there is again an abelian group action, while in the lit-only $\sigma^{+}$-game, due to the non-reversibility of moves, there is no group action at all. So the result of [26], that in terms of reachability there is essentially no difference between the regular and lit-only $\sigma^{+}$-games, may be a bit of a surprise (see Example 8). Regarding general graphs which have at least one loop, many evidences, theoretically and experimentally, support the following conjecture, the truth of which will further defend for our interest in studying the small difference between the $\sigma$-game and lit-only $\sigma$-game.

Conjecture 6. Let $G$ be a connected graph with $V(G) \cap E(G)=L \neq \emptyset$. Let $C$ be a (strongly) connected component of $\mathbb{P S}(G)$. Let $|C|=2^{r}$. If $\mathbf{0} \notin C$, then $C$ is also a strongly connected component of $\mathbb{P S}^{*}(G)$. If $\mathbf{0} \in C$, then either of the following holds: (1) $\{\mathbf{0}\}, C \backslash\left\{\mathbf{0}, \chi_{L}\right\},\left\{\chi_{L}\right\}$ are three strongly connected components of $\mathbb{P S}(G)$ and $\chi_{L} \stackrel{*}{\rightarrow}_{G}$ $\mathbf{x} \stackrel{*}{\rightarrow}_{G} \mathbf{0}$ for any $\mathbf{x} \in C$; (2) $C$ is the disjoint union of $r+1$ strongly connected components $C_{0}, C_{1}, \ldots, C_{r}$ of $\mathbb{P S}^{*}(G)$, where $C_{0}=\{\mathbf{0}\}, C_{r}=\left\{\chi_{L}\right\},\left|C_{i}\right|=\left(\begin{array}{c}r \\ i\end{array}\right)$ for $i \in\{0, \ldots, r\}$ and $\mathbf{x} \stackrel{*}{\rightarrow}_{G} \mathbf{y}$ for any $\mathbf{x} \in C_{i}, \mathbf{y} \in C_{j}$ and any $r \geq i \geq j \geq 0$.

The main discovery of [45] and this paper is that there exists a simple principle to design algorithms to play the lit-only $\sigma$-game on a graph to reach a configuration with small light number by taking advantage of our knowledge on how to play the $\sigma$-game on 
the same graph; we refer the reader to [45, Section 3] for an illustration of this strategy. We mention that the performance of these algorithms in reducing light number can be guaranteed by the existence of some local structures along with the performance of our strategy for playing the $\sigma$-game. With the help of this guiding principle, roughly speaking, our main effort is to determine these good local structures and show that they are always present in some graph classes. Computer experiments have suggested some very surprising relationship between the $\sigma$-game and lit-only $\sigma$-game [27] and so it may be worthwhile to try to understand the role of the lit-only restriction and its possible counterpart in some more general algebraic settings.

\section{Main Results}

We list below a series of observations on the difference between the minimum light numbers of the $\sigma$-game and the lit-only $\sigma$-game on several graph classes.

Example 7. Let $G$ be a graph, $L=V(G) \cap E(G)$. The famous Sutner's Theorem [1, 3, 9, 41, 47] asserts that $\chi_{L} \rightarrow_{G} \mathbf{0}$, namely $M L\left(\chi_{L}\right)=0$. We can show the stronger result that $\chi_{L} \stackrel{*}{\rightarrow}_{G} \mathbf{0}$, i.e., $M L^{*}\left(\chi_{L}\right)=0$, and will report its proof in another paper.

Example 8. Let $G$ be a graph with $V(G) \subseteq E(G)$. [26, Theorem 3] says that for any $\mathbf{x} \neq \mathbf{0}$ and $\mathbf{y} \neq \chi_{V(G)}, \mathbf{x} \rightarrow_{G} \mathbf{y}$ if and only if $\mathbf{x} \stackrel{*}{\rightarrow}_{G} \mathbf{y}$. In particular, any orbit of the $\sigma$-game on $G$ which does not contain the all-OFF configuration will also be an orbit of the lit-only $\sigma$-game on $G$. Additionally, combined with the Sutner's Theorem (Example 7), we see that $\mathcal{D}(G)=0$. Indeed, checking the proof of [26, Theorem 3] shows that a stronger result holds: If $\mathbf{x}=\mathbf{y}+\sum_{v \in S} \chi_{N_{G}(v)}, \mathbf{x} \neq \mathbf{0}$ and $\mathbf{y} \neq \chi_{V(G)}$, where $N_{G}(v) \neq \emptyset$ for any $v \in S$, then $\mathbf{x} \underset{S_{G}}{\stackrel{*}{\rightarrow}} \mathbf{y}$ holds. More results of this nature will be addressed in [27].

Let us now turn attention to trees and unicyclic graphs.

Example 9. Suppose $G$ is the 4-cycle $\left(G_{2,2}\right)$ and $\mathbf{x}$ the configuration of $G$ as depicted in Fig. 3. Then $M L^{*}(\mathbf{x})-M L(\mathbf{x})=2=\mathcal{D}(G)$.

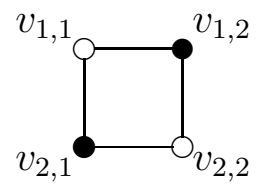

Figure 3: A configuration $\mathbf{x}$ of $G_{2,2}$ with $M L^{*}(\mathbf{x})-M L(\mathbf{x})=2$.

Example 10. Let $G$ and $\mathbf{x}$ be depicted as in Fig. 4. Then

$$
M L(G)=M L^{*}(G)=3, \quad M L_{G}(\mathbf{x})=0, \quad M L_{G}^{*}(\mathbf{x})=3 .
$$




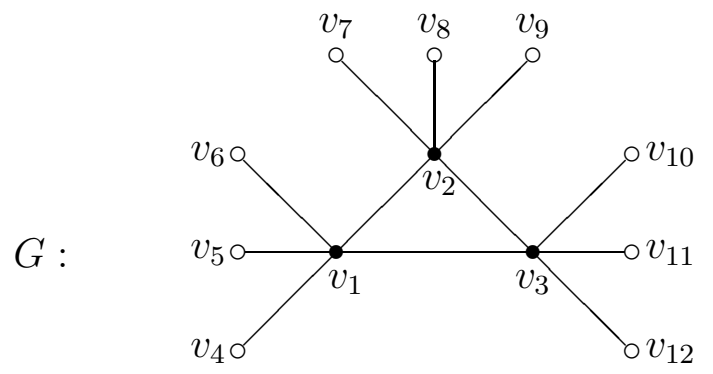

Figure 4: A loopless unicyclic graph $G$ with configuration $\mathbf{x}=\chi_{\left\{v_{1}, v_{2}, v_{3}\right\}}$.

Theorem 11. [32, Theorem 1.9] If $G$ is a tree with a perfect matching, then $M L_{G}^{*}(\mathbf{x})=1$ and $M L_{G}(\mathbf{x})=0$ for any $\mathbf{x} \in \mathbb{F}_{2}^{V(G)} \backslash\{\mathbf{0}\}$.

Theorem 12. [45, Theorem 14] If $G$ is a pseudo-tree, then the inequality $\mathcal{D}(G) \leq 2$ holds and the upper bound 2 is sharp.

Theorem 13. Let $G$ be a graph. If $\mathcal{S}(G)$ is unicyclic, then $\mathcal{D}(G) \leq 3$ and the upper bound 3 is tight.

Similar to an earlier conjecture for trees [45, Conjecture 7], we propose the next one on unicyclic graphs.

Conjecture 14. Let $G$ be a graph such that $\mathcal{S}(G)$ is unicyclic. Then $M L^{*}(G)-M L(G) \leq$ 2 .

Let us remark that Nath and Sarma found a nice combinatorial characterization for a tree or a unicyclic graph to have a nonsingular adjacency matrix [37, Theorems 3.1, 3.4]. Their proof is given for the real field but can be checked to be valid over the binary field as well. As suggested by Theorem 11, it might be a good idea to tackle Conjecture 14 firstly for those nonsingular loopless graphs as in that case we have a nice equivalent representation of the lit-only $\sigma$-game $[32,39]$, of which we give a brief introduction at the end of Section 3.

For any $n \geq 3$, the flower graph $\mathfrak{F}_{n}$ of order $2 n$ is the graph with vertex set $\left\{v_{1}, \ldots, v_{n}\right.$, $\left.u_{1}, \ldots, u_{n}\right\}$ and edge set $\left\{v_{1} v_{2}, v_{2} v_{3}, \ldots, v_{n-1} v_{n}, v_{n} v_{1}\right\} \cup\left\{v_{1} u_{1}, v_{2} u_{2}, \ldots, v_{n} u_{n}\right\}$, i.e., the $n$ cycle with a leaf at each vertex; see Fig 5 . The following observation says that the bound in Conjecture 14 is best possible, if it is true.

Theorem 15. For any $n \geq 3$ we have $M L\left(\mathfrak{F}_{n}\right)=0, \mathcal{D}\left(\mathfrak{F}_{n}\right)=M L^{*}\left(\mathfrak{F}_{n}\right)=2$.

To be compared with Example 10 and Theorem 15, let us also take a look at those graphs obtained by 'planting' a pseudo-tree with at least two branch vertices on a connected graph. If we relax the condition of Theorem 16 a bit to allow that $G\left[V_{1}\right]$ has at most one branch vertex, then its conclusion may become false; see Example 10 (Fig. 4). 


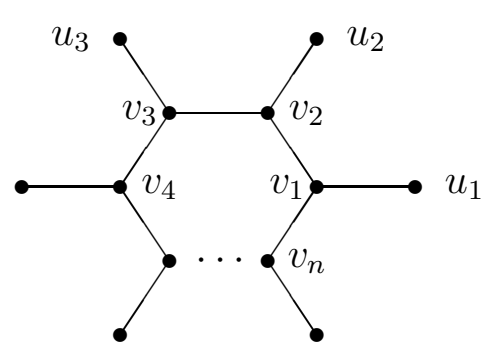

Figure 5: The flower graph $\mathfrak{F}_{n}$.

Theorem 16. Let $G$ be a graph with $V(G)=V_{1} \cup V_{2}$ and $V_{1} \cap V_{2}=\{u\}$. Suppose that $G\left[V_{1}\right]$ is a pseudo-tree which contains at least two branch vertices and $G\left[V_{2}\right]$ is connected. If there is no edge in $G$ which intersects both $V_{1} \backslash\{u\}$ and $V_{2} \backslash\{u\}$, then $\mathcal{D}(G) \leq 2$ and this upper bound is sharp.

In the study of the $\sigma$-game, the most widely investigated graph class is that of the 2-dimensional grid graphs. Let us report some results on grid graphs and the lit-only $\sigma$-game.

Theorem 17. [25, Theorem 15] The parameter $M L\left(G_{m, n}\right)$ equals $\frac{d}{2}+1$ if $d \equiv 2(\bmod 4)$ and $\left\lceil\frac{d}{2}\right\rceil$ otherwise, where $d=\operatorname{gcd}(m+1, n+1)-1$.

Theorem 18. [25, Theorem 16] $M L^{*}\left(G_{m, n}\right) \leq \min (m, n)$.

The next result strengthens Theorem 18 a little bit.

Theorem 19. If $\mathcal{S}(G)=G_{m, n}$, then $M L^{*}(G) \leq \min (m, n)$.

Let $G$ be a graph. For any configuration $\mathbf{x} \in \mathbb{F}_{2}^{V(G)}$, we say that a vertex $v$ is admissible in $\mathbf{x}$ provided either $v \in V(G) \cap E(G)$ and $\mathbf{x}(v)=0$ or $v \in V(G) \backslash E(G)$ and $\mathbf{x}(v)=1$. If $v$ is admissible in $\mathbf{x}$, a reverse-lit-only move at $v$, or a reverse-move at $v$ in short, transforms $\mathbf{x}$ to $\mathbf{x}+\chi_{N_{G}(v)}$. It is clear that a sequence of reverse-lit-only moves bring $\mathbf{x}$ to $\mathbf{y}$ if and only if $\mathbf{y} \stackrel{*}{\rightarrow}_{G} \mathbf{x}$ (just reverse the sequence of toggles). So, the toggling game specified by the reverse-lit-only moves will be called the reverse game of the lit-only $\sigma$-game.

By examining the reachability of the phase space of the lit-only $\sigma$-game in view of both the lit-only moves and reverse-lit-only moves, we obtain the following result, which may be a bit surprising at first sight.

Theorem 20. Let $G$ be a graph with $\mathcal{S}(G)=G_{m, n}$ for integers $m \geq 2$ and $n \geq 3$. Suppose $L=V(G) \cap E(G) \neq \emptyset$ and $Q$ is a subset of $V(G)$. If $\mathbf{x}$ is any non-zero configuration in $\mathbb{F}_{2}^{V(G)}$ and $\mathbf{y}=\mathbf{x}+\sum_{v \in Q} \chi_{N_{G}(v)} \neq \chi_{L}$, then $\mathbf{x}{\underset{Q}{G}}_{G} \mathbf{y}$.

From Fig. 16 in Appendix A, we see that the configuration (0111) will go back to itself by regular togglings at vertices 1,2 and 3 but it is not true that $(0111) \underset{\{1,2,3\}}{\stackrel{*}{\longrightarrow}}(0111)$. Hence the conclusion of Theorem 20 does not hold when $m=n=2$. 
Theorem 21. Let $G$ be a graph with $\mathcal{S}(G)=G_{m, n}$ for two integers $m, n \geq 2$. Suppose $L=V(G) \cap E(G) \neq \emptyset$. For any $\mathbf{x} \in \mathbb{F}_{2}^{V(G)} \backslash\{\mathbf{0}\}$ and $\mathbf{y} \in \mathbb{F}_{2}^{V(G)} \backslash\left\{\chi_{L}\right\}$, if $\mathbf{x} \rightarrow_{G} \mathbf{y}$ then $\mathbf{x} \stackrel{*}{\rightarrow}_{G} \mathbf{y}$.

Theorem 22. Let $G$ be a graph with $V(G) \cap E(G) \neq \emptyset$ and $\mathcal{S}(G)=G_{m, n}$ for $m, n \geq 2$. Then $\mathcal{D}(G)=0$.

Note that a consequence of Theorem 19 is that $\mathcal{D}(P) \leq 1$ for any pseudo-path $P$. A deeper understanding of the inequality $\mathcal{D}(P) \leq 1$ is embodied in the next two theorems.

Theorem 23. Let $P$ be a pseudo-path with at least one loop and $\mathbf{x}$ a configuration of $P$. If $\mathbf{x} \rightarrow \mathbf{0}$ then $\mathbf{x} \stackrel{*}{\rightarrow} \mathbf{0}$.

Theorem 24. Let $\mathbf{x}$ be a configuration on a pseudo-path $P$ where $P \neq \mathcal{S}(P)=\left[v_{1}, v_{2}\right.$, $\left.\ldots, v_{n}\right]$. If $\mathbf{x}$ cannot be reduced to $\mathbf{0}$ by regular togglings then it can be reduced by lit-only togglings to just $v_{1} O N$ and to just $v_{n} O N$.

Let us mention that the lit-only $\sigma$-game on a pseudo-path can be analyzed even more satisfactorily than as reported in Theorems 23 and 24 due to the fact that a path is a line graph and the corresponding result will be reported in a separate paper on line graphs and related structures.

Owing to Example 9 (Fig. 3), we see that the next result is best possible in some sense.

Theorem 25. For any positive integers $m$ and $n, \mathcal{D}\left(G_{m, n}\right) \leq 2$.

When $m$ and $n$ are at least 4 , the ensuing result is another slight improvement of Theorem 18.

Theorem 26. Let $m$ and $n$ be positive integers and let $d=\operatorname{gcd}(m+1, n+1)-1$. Then $M L^{*}\left(G_{m, n}\right) \leq \frac{d}{2}+3$ if $d \equiv 2(\bmod 4)$ and $M L^{*}\left(G_{m, n}\right) \leq\left\lceil\frac{d}{2}\right\rceil+2$ otherwise.

Proof. This follows from Theorems 3, 17 and 25.

Theorem 27. [25, Theorem 17] The parameter $M L^{*}\left(G_{m, 2}\right)$ is equal to 2 if $m \equiv 2(\bmod 3)$ and is equal to 1 otherwise.

Let $G_{m, n}^{+}$be the graph obtained from $G_{m, n}$ by attaching loops everywhere. Goldwasser, Klostermeyer and Trapp [21] use Fibonacci polynomials to deduce the number of orbits in the $\sigma$-game on $G_{m, n}^{+}$. This also gives some information on the lit-only $\sigma$-game on $G_{m, n}^{+}$ in view of Example 8.

We recall some technical lemmas in the next section. After that, we will devote ourselves to proving Theorems 13, 15, 16, 19, 20, 21, 22, 23, 24 and 25. In the course of presenting the proofs, we will also collect some relevant results and provide some discussion and problems. 


\section{Preliminaries}

For any two integers $n, k \geq 1$, the rake with $k$ teeth $w_{1}, \ldots, w_{k}$ and an $n$-handle $v_{1}, \ldots, v_{n}$ is the graph $P_{n, k}$ with $V\left(P_{n, k}\right)=\left\{v_{1}, v_{2}, \ldots, v_{n}, w_{1}, \ldots, w_{k}\right\}$ and $E\left(P_{n, k}\right)=\left\{v_{1} v_{2}, v_{2} v_{3}\right.$, $\left.\ldots, v_{n-1} v_{n}, v_{n} w_{1}, \ldots, v_{n} w_{k}\right\}$; see Fig. 6. The top of the rake is $v_{1}$ and all the other vertices are called the common vertices. When $k=1, P_{n, k}$ is just an $(n+1)$-path one of whose two leaves is specified as the top. When $n=1, P_{n, k}$ is also known as a $k$-star.

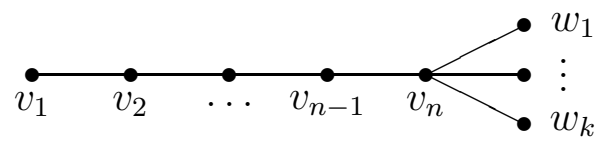

Figure 6: The rake $P_{n, k}$ with $k$ teeth and an $n$-handle.

What come next are some preliminary results prepared in [45]. The reader can try to prove them as a warm-up and a test of his/her understanding of the basic strategy introduced in [45, Section 3].

Lemma 28. [45, Lemma 18] Let $G$ be a connected graph and $\mathbf{x} \in \mathbb{F}_{2}^{V(G)} \backslash\{\mathbf{0}\}$. Suppose $a$ and $b$ are two vertices of $G$ satisfying $N_{G}(a) \neq N_{G}(b)$. Then, there is $\mathbf{y} \in \mathbb{F}_{2}^{V(G)}$ such that $\mathbf{y}(a) \neq \mathbf{y}(b)$ and $\mathbf{x} \stackrel{*}{\rightarrow}_{G} \mathbf{y}$.

Lemma 29. [45, Lemma 19] Let $G$ be a graph, $a, b \in V(G)$, ab $\notin E(G), c \in N_{G}(a) \cap$ $N_{G}(b)$. Let $S \subseteq V(G) \backslash\left(N_{G}(a) \cup N_{G}(b)\right)$ such that $G[S \cup\{c\}]$ is connected. Assume that $\mathbf{x}$ and $\mathbf{y}$ are two configurations of $G$ such that $\mathbf{x}=\mathbf{y}+\sum_{v \in Q} \chi_{N_{G}(v)}$ for some $Q \subseteq V(G)$ and $\chi_{N_{G}(v)} \neq \mathbf{0}$ for any $v \in Q$. If $\mathbf{x}(a) \neq \mathbf{x}(b)$, then there exists $R \subseteq V(G) \backslash(S \cup\{c\})$ such that $\mathbf{x} \underset{Q \triangle R}{\stackrel{*}{\longrightarrow}} \mathbf{z}=\mathbf{y}+\sum_{v \in R} \chi_{N_{G}(v)}$ and $\mathbf{z}(a) \neq \mathbf{z}(b)$.

Lemma 30. [45, Lemma 22] Let $G$ be a connected graph, $c \in V(G)$, and $\mathbf{x} \in \mathbb{F}_{2}^{V(G)}$. Suppose that $U$ and $W$ are two components of $G-c$. Further assume that the shadow graphs of $G[\{c\} \cup U]$ and $G[\{c\} \cup W]$ are both rakes with $c$ being its top. If there are $u \in U$ and $w \in W$ such that either $N_{G}(u) \neq N_{G}(w)$ or $\mathbf{x}(u) \neq \mathbf{x}(w)$, then $M L_{G}^{*}(\mathbf{x})-M L_{G}(\mathbf{x}) \leq$ 2. Furthermore, $M L_{G}^{*}(\mathbf{x})-M L_{G}(\mathbf{x}) \leq 1$ if $|U|=|W|=1$.

Finally, let us discuss an interesting invariant of the lit-only $\sigma$-game [39], following the nice discovery of $\left[32\right.$, Section 4]. Let $G$ be a graph and let $E_{v}=\chi_{v} \chi_{v}^{\top}$ be the $|V(G)| \times|V(G)|$ matrix with a lone 1 on its $(v, v)$-position. Let $A$ be the adjacency matrix of $G$ and let $I=\sum_{v \in V(G)} E_{v}$ be the identity matrix. In the lit-only $\sigma$-game on $G$, a lit-only move at $v$ transforms a configuration $\mathbf{x}$ to $\mathbf{y}$ if and only if

$$
\mathbf{y}=\left(I+A E_{v}\right) \mathbf{x}
$$

If $A$ is nonsingular, by multiplying $A^{-1}$ on the left of both sides of Eq. (1), we see that a lit-only move at $v$ brings $\mathbf{x}$ to $\mathbf{y}$ if and only if

$$
A^{-1} \mathbf{y}=\left(I+E_{v} A\right) A^{-1} \mathbf{x} .
$$

THE ELECTRONiC JOURNAL OF COMBINATORICS 18 (2011), \#P214 
For any configuration $\mathbf{x}$ of $G$, define $\mathcal{R}_{G}(\mathbf{x})=\sum_{v \in V(G)} \mathbf{x}(v)+\sum_{u v \in E(G) \backslash V(G)} \mathbf{x}(u) \mathbf{x}(v)$. If $G$ is loopless, it is easy to check that $\mathcal{R}_{G}(\mathbf{z})=\mathcal{R}_{G}\left(\left(I+E_{v} A\right) \mathbf{z}\right)$ holds for any vertex $v$ and any configuration $\mathbf{z}$. Combined with the relationship between a lit-only move and Eq. (2), we arrive at the conclusion

$$
\mathbf{x} \stackrel{*}{\rightarrow}_{G} \mathbf{y} \Rightarrow \mathcal{R}_{G}\left(A^{-1} \mathbf{x}\right)=\mathcal{R}_{G}\left(A^{-1} \mathbf{y}\right)
$$

whenever $G$ is both loopless and nonsingular.

\section{Unicyclic graphs}

Lemma 31. Let $G$ be a graph. If $\mathcal{S}(G)$ is a cycle $\left\langle u_{1}, u_{2}, \ldots, u_{s}\right\rangle$, then $M L^{*}(G) \leq 2$.

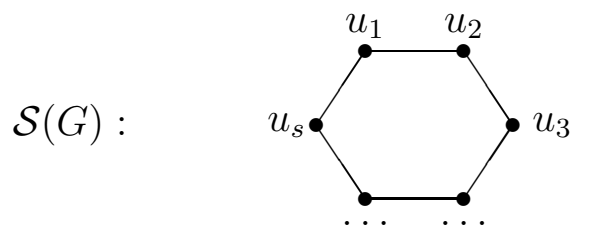

Proof. Let $\mathrm{x}$ be any configuration of $G$. Let $G^{\prime}$ be the graph obtained from $G$ by deleting the edge $u_{1} u_{s}$. Lemma 1 applied to $G^{\prime}$ and $\mathbf{x}$ says that there is a series of valid moves inside $V(G) \backslash\left\{u_{1}\right\}$ which transforms $\mathbf{x}$ to a configuration $\mathbf{y}$ with at most one ON vertex. The same series of moves are still valid on $G$ and the resulting configuration $\mathbf{y}^{\prime}$ can differ with $\mathbf{y}$ only at vertex $u_{1}$ and this possible difference can only be caused by valid moves at $u_{s}$. This gives $M L_{G}^{*}(\mathbf{x}) \leq 2$, completing the proof.

As suggested by the above proof, a natural question is as follows: Given any rooted tree, can we play the lit-only $\sigma$-game on it to reduce the light number of any starting configuration to at most two without invoking any move at the root? If this is always possible, we can immediately get a proof of Theorem 13 analogous to that of Lemma 31. Unfortunately, this is too good to be expected as can be seen from the all-ON configuration of the $k$-star for $k>2$ with its top as the specified root.

To investigate more general unicyclic graphs, we need the following "rooted" version of [45, Lemma 17].

Lemma 32. For any tree $T$ with at least two vertices and a specified vertex $r$, one of the following holds:

(a) $T$ is a rake with $r$ being its top;

(b) There is a vertex $v \in V(T)$ for which $T-v$ contains two components $U$ and $W$ such that $r \notin U \cup W$ and both $T[\{v\} \cup U]$ and $T[\{v\} \cup W]$ are rakes with $v$ being the top and $|W| \geq 2$.

Proof. We distinguish three cases. 
CASE 1: $V(T) \backslash\{r\}$ does not contain any branch vertex.

There are three possibilities: Either $T-r$ has only one component, or has more than one component but each of them is of size one, or contains at least two components and the biggest size of these components is greater than one. In the first case, $T$ is an $n$-path for some $n \geq 2$ and so (a) holds; in the middle case, $T$ is a $k$-star for some $k \geq 2$ and so (a) holds; in the final case, we choose a largest component of $T-r$ as $W$ and any other component as $U$ and set $v=r$, establishing (b).

CASE 2: The maximum number of branch vertices inside $V(T) \backslash\{r\}$ which can appear in a common path starting from $r$ is 1 .

If there is a branch vertex $v \in V(T) \backslash\{r\}$ and a component $W$ of $T-v$ such that $r \notin W$ and $|W| \geq 2$, then we take $U$ to be any component of $T-v$ such that $U \neq W$ and $r \notin U$ and then yield (b).

In the remaining case, each component of $T-r$ is a rake, one of them containing a branch vertex and hence of size at least 3, and so we either have (a) or can choose $v=r$ to obtain (b).

CASE 3: The maximum number of branch vertices inside $V(T) \backslash\{r\}$ which can appear simultaneously in a path starting from $r$ is $k \geq 2$.

Choose a path in $T$ with $r$ being one endpoint and which passes through as many branch vertices of $T$ as possible. Suppose that after starting from $r$ in this path, the branch vertices appearing after $r$ are $w_{1}, \ldots, w_{k}$ in that order. If this path can be chosen such that $T-w_{k}$ has a component $W$ which does not contain $r$ and has size at least two, we then put $v=w_{k}$ and take $U$ to be any component of $T-v$ which is not $W$ and does not contain $r$. Otherwise, we set $v=w_{k-1}$, let $W$ be the component of $T-v$ that contains $w_{k}$, and let $U$ be any component of $T-w_{k-1}$ subject to $U \neq W$ and $r \notin U$. It is easy to check that the requirements for (b) are fulfilled in both situations.

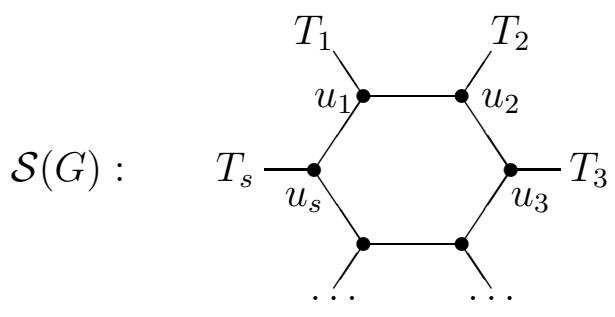

Figure 7: A unicyclic graph.

Proof of Theorem 13. In view of Example 10, what remains to do is to show $M L_{G}^{*}(\mathbf{x})-$ $M L_{G}(\mathbf{x}) \leq 3$ for every $\mathbf{x} \in \mathbb{F}_{2}^{V(G)}$.

Suppose that $\mathcal{S}(G)$ is obtained from a cycle $\left\langle u_{1}, u_{2}, \ldots, u_{s}\right\rangle, s \geq 3$, by attaching a tree $T_{i}$ at $u_{i}$ for $i=1, \ldots, s$; see Fig. 7 . We specify $u_{i}$ as the root of $T_{i}$ for each index $i$. Let us 
then play the lit-only $\sigma$-game on $G$ with the initial configuration x. By Lemma 32, the following four cases are exhaustive.

CAse 1: $V\left(T_{i}\right)=\left\{u_{i}\right\}$ for every $i \in\{1, \ldots, s\}$.

In this case, Lemma 31 leads to $M L_{G}^{*}(\mathbf{x})-M L_{G}(\mathbf{x}) \leq 2$.

CASE 2: There exists an index $i$ such that the tree $T_{i}$ rooted at $u_{i}$ has more than one vertex and statement (b) in Lemma 32 holds for it.

It follows from Lemma 30 that $M L_{G}^{*}(\mathbf{x})-M L_{G}(\mathbf{x}) \leq 2$.

CASE 3: There exists an index $i$ such that the tree $T_{i}$ rooted at $u_{i}$ is a rake with $u_{i}$ being the top such that either it has two teeth which are in different states in $\mathbf{x}$ or it has at least two teeth and at least one of them has a loop.

Taking $c$ to be the vertex on the handle of $T_{i}$ which is adjacent to all the teeth of $T_{i}$, we deduce from Lemma 30 that $M L_{G}^{*}(\mathbf{x})-M L_{G}(\mathbf{x}) \leq 1$.

CASE 4: There exists an index $i$ such that the tree $T_{i}$ rooted at $u_{i}$ is a rake with $u_{i}$ being the top and all its teeth are in the same state in $\mathbf{x}$ and do not have loops. Without loss of generality, suppose $i=1$ and $T_{1}$ is given as in Fig. 6 where $u_{1}=v_{1}$ is the top. Let $P=\left\{v_{1}, v_{2}, \ldots, v_{n}\right\}$.

CASE 4.1: For every $\mathbf{y}$ such that $\mathbf{x} \stackrel{*}{\rightarrow}_{G} \mathbf{y}$ we have $\operatorname{supp}(\mathbf{y}) \backslash V\left(T_{1}\right) \neq \emptyset$.

Since $N_{G}\left(w_{1}\right)=\cdots=N_{G}\left(w_{k}\right)$, we can assume that there is a configuration $\mathbf{z}$ satisfying $L(\mathbf{z})=M L_{G}(\mathbf{x})$ and $\mathbf{z}=\mathbf{x}+\sum_{v \in U} \chi_{N(v)}+\sum_{v \in W} \chi_{N(v)}$ where $U \subseteq P \cup\left\{w_{1}\right\}$ and $W \subseteq V(G) \backslash V\left(T_{1}\right)$.

Let $v^{\prime}$ be the vertex in $U$, if any, which is farthest from $v_{1}$ and let $S$ be the union of $V(G) \backslash V\left(T_{1}\right)$ and the set of vertices on the unique path connecting $v^{\prime}$ and $v_{1}$. By assumption, there must exist a vertex $u \in S$ and a path connecting $u$ and $v^{\prime}$ on which the only ON vertex is $u$. Pushing all the vertices on this path from $u$ to $v^{\prime}$ in that order brings us to a new configuration $\mathbf{x}^{\prime}$ such that $\mathbf{z}=\mathbf{x}^{\prime}+\sum_{v \in U^{\prime}} \chi_{N(v)}+\sum_{v \in W^{\prime}} \chi_{N(v)}$ where $U^{\prime} \subseteq$ $P \cup\left\{w_{1}\right\}, W^{\prime} \subseteq V(G) \backslash V\left(T_{1}\right)$, and either $U^{\prime}=\emptyset$ or distance $\left(U^{\prime}, w_{1}\right)>\operatorname{distance}\left(U, w_{1}\right)$. Continuing in this way, we know that there exists $\mathbf{y} \in \mathbb{F}_{2}^{V(G)}$ such that $\mathbf{x} \stackrel{*}{\rightarrow}_{G} \mathbf{y}$ and $\mathbf{z}=\mathbf{y}+\sum_{v \in R} \chi_{N(v)}$ for some $R \subseteq V(G) \backslash V\left(T_{1}\right)$.

The graph $G\left[V(G) \backslash V\left(T_{1}\right)\right]$ is a pseudo-tree and so an application of Theorem 12 shows that we can start from $\mathbf{y}$ and execute a series of valid moves inside $V(G) \backslash V\left(T_{1}\right)$ and get to a configuration $\mathbf{z}^{\prime}$ such that $\left|\operatorname{supp}\left(\mathbf{z}^{\prime}\right) \backslash V\left(T_{1}\right)\right| \leq\left|\operatorname{supp}(\mathbf{z}) \backslash V\left(T_{1}\right)\right|+2$. Note that $\operatorname{supp}\left(\mathbf{z}^{\prime}\right) \cap V\left(T_{1}\right) \subseteq\left\{u_{1}\right\} \cup\left(\operatorname{supp}(\mathbf{z}) \cap V\left(T_{1}\right)\right)$ as moves in $V(G) \backslash V\left(T_{1}\right)$ cannot affect the state of any vertex in $V\left(T_{1}\right) \backslash\left\{u_{1}\right\}$. This then exhibits that $M L_{G}^{*}(\mathbf{x}) \leq L\left(\mathbf{z}^{\prime}\right) \leq$ $L(\mathbf{z})+2+1=M L_{G}(\mathbf{x})+3$, as required.

CASE 4.2: There is $\mathbf{y}$ such that $\mathbf{x} \stackrel{*}{\rightarrow}_{G} \mathbf{y}$ and $\operatorname{supp}(\mathbf{y}) \subseteq V\left(T_{1}\right)$. 
CASE 4.2.1: If $\mathbf{y}\left(w_{1}\right)=\cdots=\mathbf{y}\left(w_{k}\right)=0$, we apply Lemma 1 on $G[P]$ and find that there is a sequence of valid moves inside $v_{1}, \ldots, v_{n-1}$ that transforms $\mathbf{y}$ to $\mathbf{z}$ satisfying $|\operatorname{supp}(\mathbf{z}) \cap P| \leq 1$. But the only vertices in $V(G) \backslash P$ which have a possibility to be assigned the ON state are $u_{2}$ and $u_{s}$. This gives $M L_{G}^{*}(\mathbf{x}) \leq L(\mathbf{z}) \leq 1+2=3$.

CASE 4.2.2: If Case 4.2.1 does not happen, we must have $\mathbf{y}\left(w_{1}\right)=\cdots=\mathbf{y}\left(w_{k}\right)=1$. If $k=1$ and there is a loop at $w_{1}$, then a valid move at $w_{1}$ takes us back to Case 4.2.1. For the remaining case, we will have $N_{G}\left(w_{i}\right)=\left\{v_{n}\right\}$ for any $i \in\{1, \ldots, k\}$. If $\mathbf{y}\left(v_{n}\right)=1$, we make a valid move at $v_{n}$ and otherwise we make two consecutive valid moves at $w_{1}$ and $v_{n}$ in that order. In both cases we will either be reduced to Case 4.2 .1 when $n>1$ or else reach a configuration whose only $\mathrm{ON}$ vertices are $u_{s}, u_{2}$ and possibly $u_{1}$, implying that $M L_{G}^{*}(\mathbf{x}) \leq 3$.

Before presenting the proof of Theorem 15, let us prepare a technical lemma on the flower graph.

Lemma 33. Take two subsets $I$ and $J$ of $\{1, \ldots, n\}$. Then there exists a subset $I^{\prime}$ of $I$ such that $\sum_{i \in I} \chi_{N\left(v_{i}\right)}+\sum_{j \in J} \chi_{N\left(u_{j}\right)} \stackrel{*}{\rightarrow} \mathfrak{F}_{n} \sum_{i \in I^{\prime}} \chi_{N\left(u_{i}\right)}+\sum_{j \in J} \chi_{N\left(u_{j}\right)}$.

Proof. Let $\mathbf{x}=\sum_{i \in I} \chi_{N\left(v_{i}\right)}+\sum_{j \in J} \chi_{N\left(u_{j}\right)}$. It suffices to prove for any $i \in I$ that either $\mathbf{x} \stackrel{*}{\rightarrow}_{\mathfrak{\mho}_{n}} \mathbf{x}+\chi_{N\left(v_{i}\right)}$ or $\mathbf{x} \stackrel{*}{\rightarrow}_{\mathfrak{\mho}_{n}} \mathbf{x}+\chi_{N\left(v_{i}\right)}+\chi_{N\left(u_{i}\right)}$. Indeed, $i \in I$ means that $\mathbf{x}\left(u_{i}\right)=1$ and so either a move at $v_{i}$ or successive moves at $u_{i}$ and $v_{i}$ will be allowed and that gives the result.

Proof of Theorem 15. Let $A_{n}$ be the adjacency matrix of $\mathfrak{F}_{n}$. Since there is a unique matching between the leaves and the vertices on the cycle, it is immediate that there is only one nonzero term in the expansion of $\operatorname{det} A_{n}$ and hence $M L\left(\mathfrak{F}_{n}\right)=0$.

We next show that $M L^{*}\left(\mathfrak{F}_{n}\right) \leq 2$. Given a configuration $\mathbf{x} \neq \mathbf{0}$, we want to show $M L^{*}(\mathbf{x}) \leq 2$. Note that $M L\left(\mathfrak{F}_{n}\right)=0$ guarantees $\mathbf{x} \rightarrow \mathbf{0}$. Taking $(a, b, c)=\left(v_{2}, u_{1}, v_{1}\right)$, $S=V(G) \backslash\left\{v_{1}, v_{2}, v_{3}, u_{1}, u_{2}, u_{3}\right\}$ and $G=\mathfrak{F}_{n}$, Lemma 28 along with Lemma 29 now implies that there is a configuration $\mathbf{y}_{1}$ such that $\mathbf{x} \stackrel{*}{\rightarrow} \mathfrak{F}_{n} \mathbf{y}_{1}$, and $\mathbf{y}_{1}=\sum_{w \in I_{1}} \chi_{N(w)}$ for some $I_{1} \subset\left\{v_{2}, v_{3}, u_{1}, u_{2}, u_{3}\right\}$. Applying Lemma 33 we find that there is a subset $I_{2}$ of $\{1,2,3\}$ such that

$$
\mathbf{x} \stackrel{*}{\rightarrow}_{\mathfrak{F}_{n}} \mathbf{y}_{1} \stackrel{*}{\rightarrow}_{\mathfrak{F}_{n}} \mathbf{y}_{2}
$$

where $\mathbf{y}_{2}=\sum_{i \in I_{2}} \chi_{N\left(u_{i}\right)}$. If $I_{2} \neq\{1,2,3\}$, then $L\left(\mathbf{y}_{2}\right) \leq 2$ and so Eq. (4) already demonstrates $M L^{*}(\mathbf{x}) \leq 2$. If $I_{2}=\{1,2,3\}$, then $\mathbf{y}_{2} \underset{v_{2}}{\stackrel{*}{\longrightarrow}} \chi_{\mathfrak{F}_{n}} \chi_{u_{2}}+\chi_{v_{2}}$, hence yielding $M L^{*}\left(\mathfrak{F}_{n}\right) \leq 2$ as before.

It remains to prove $M L^{*}\left(\mathfrak{F}_{n}\right) \geq 2$. For $i \in\{1,2, \ldots, n\}$, we have $A_{n}^{-1} \chi_{v_{i}}=\chi_{u_{i}}$ and $A_{n}^{-1} \chi_{u_{i}}=\chi_{v_{i}}+\chi_{u_{i-1}}+\chi_{u_{i+1}}$ where the subscripts $i-1$ and $i+1$ should be read modulo $n$. Therefore, we find that for any $w \in V\left(\mathfrak{F}_{n}\right), \mathcal{R}\left(A_{n}^{-1} \chi_{w}\right)=1$. In view of Eq. (3) and the fact that the all-OFF configuration cannot be reached in the lit-only $\sigma$-game on a loopless graph from any other configuration, this then leads to $M L^{*}(\mathbf{x}) \geq 2$ for any nonzero configuration $\mathbf{x}$ with $\mathcal{R}\left(A_{n}^{-1} \mathbf{x}\right)=0$ and it then follows $M L^{*}\left(\mathfrak{F}_{n}\right) \geq 2$, as wanted. 


\section{Graphs containing a pseudo-tree with at least two branch vertices}

Let $H$ be a graph and $u$ one of its vertices. Adapting a bit a definition made by Nylen [38, p. 309], we say that a vertex $v$ of $G$ is appropriate for $u$ provided there are at least two components of $H[V(H) \backslash\{v\}]$ which do not contain $u$ and are pseudo-rakes whose tops are the only vertices in the components that are adjacent to $v$.

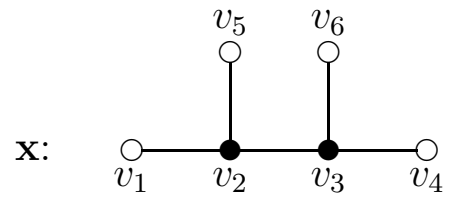

Figure 8: $M L^{*}(\mathbf{x})-M L(\mathbf{x})=2([45$, Example 9$])$.

Proof of Theorem 16. The sharpness of the bound is seen from [45, Example 9]; we reproduce this example in Fig. 8. It then remains to establish the asserted bound. By virtue of Lemma 30, the only case that we need to worry about is the situation where every component of $G[V(G) \backslash\{v\}]$ that is a pseudo-rake contains exactly one vertex, where $v$ is any appropriate vertex of $G$ for $u$. We end the proof by deriving a contradiction under the assumption that this case happens.

Let $H$ be the shadow graph of $G\left[V_{1}\right]$ and let $k$ be the maximum number such that there is a path in $G\left[V_{1}\right]$ passing through $k$ branch vertices of $H$ and having $u$ as one endpoint. Since $G\left[V_{1}\right]$ has at least two branch vertices, we immediately know that $k \geq 2$. Now take a path in $G\left[V_{1}\right]$ starting from $u$ such that the branch vertices $v_{1}, v_{2}, \ldots, v_{k}$ appear in that order along the path. It is not difficult to see that $v_{k-1}$ is appropriate for $u$ but the component of $G\left[V(G) \backslash\left\{v_{k-1}\right\}\right]$ containing $v_{k}$ is a pseudo-rake with more than one vertices, yielding the desired contradiction.

We remark that a shorter proof of Theorem 16 follows from an application of Lemma 30 and Lemma 32, though the proof of Lemma 32 itself is not short.

\section{Grid graphs with loops attached}

To begin with, let us prove a result on general pseudo-grid graphs, namely Theorem 19 . The idea of our proof is similar to the proof of Goldwasser and Klostermeyer [25] for Theorem 18 but not the same.

Proof of Theorem 19. Without loss of generality, we suppose that $m \leq n$. We only give the proof for $m=2$; the same idea works if $m>2$. We play the lit-only $\sigma$-game on $G$ with any initial configuration. We label the elements of $V(G)=V\left(G_{2, n}\right)$ as in Fig. 9 . For each $k=1,2, \ldots, 2 n-2$, as long as one of $\left\{v_{k+2}, v_{k+3}, \ldots, v_{2 n}\right\}$ is $\mathrm{ON}$, we execute a 
sequence of valid moves inside $\left\{v_{k+2}, v_{k+3}, \ldots, v_{2 n}\right\}$ to turn $v_{k}$ OFF. Finally, at most two vertices are $\mathrm{ON}$.

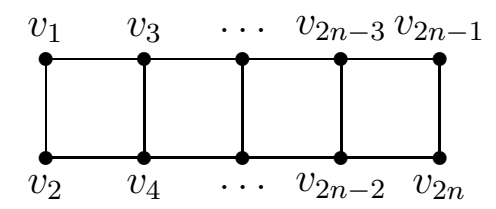

Figure 9: $G_{2, n}$ with vertex set $\left\{v_{1}, \ldots, v_{2 n}\right\}$.

Lemma 34. Let $G$ be a connected graph. For any vertex $u$ of $G$ and any configuration $\mathbf{x}$ of $G$ in which we can find at least one admissible vertex, there is a configuration $\mathbf{y}$ such that $\mathbf{y} \stackrel{*}{\rightarrow}_{G} \mathbf{x}$ and $u$ is admissible in $\mathbf{y}$.

Proof. If $w$ is not admissible but it has an admissible neighbor $w^{\prime}$, then a reverse-lit-only move at $w^{\prime}$ makes $w$ admissible.

Lemma 35. Let $G$ be a graph with $\mathcal{S}(G)=G_{m, n}$ where $m \geq 2$ and $n \geq 3, L=V(G) \cap$ $E(G), u \notin L, v \in L, u v \in E(G), \mathbf{y} \in \mathbb{F}_{2}^{V(G)}, \mathbf{y} \neq \chi_{L}$. Then there exists $\mathbf{z} \in \mathbb{F}_{2}^{V(G)}$ such that $\mathbf{z} \stackrel{*}{\rightarrow}_{G} \mathbf{y}$ and $\mathbf{z}(u)=\mathbf{z}(v)=0$.

Proof. Our task is to show that we can make a sequence of reverse-lit-only moves to go from $\mathbf{y}$ to a configuration $\mathbf{z}$ such that $\mathbf{z}(u)=\mathbf{z}(v)=0$.

Since $\mathbf{y} \neq \chi_{L}$, at least one vertex is admissible in $\mathbf{y}$. By Lemma 34, we can start from the initial configuration $\mathbf{y}$ and play the reverse game to reach a configuration where $u$ is admissible. If $v$ is not admissible, one more reverse-move at $u$ will make both $u$ and $v$ admissible, namely $u$ is ON and $v$ is OFF.

For the current configuration, if $u$ has an admissible neighbor $w \neq v$, then after a reverse-move at $w$, both $u$ and $v$ are OFF. Similarly, if $v$ has an inadmissible neighbor $w$, then after consecutive reverse-moves at $v$ and $w$, both $u$ and $v$ are OFF. So we can assume that no vertex from $N_{G}(u) \backslash\{v\}$ is admissible and each vertex from $N_{G}(v)$ is admissible.

CASE 1: Both $u$ and $v$ are corner vertices of $G$. Because of our assumption that $m \geq 2$ and $n \geq 3, \mathcal{S}(G)$ has an induced subgraph $H$ as shown in Fig. 10. If $w_{3}$ is not admissible, after two consecutive reverse-moves at $u$ and $w_{4}, w_{3}$ becomes admissible and the states of $u$ and $v$ are unchanged. Then either $w_{3}, w_{2}, w_{1}$ or $w_{2}, w_{1}$ is a sequence of reverse-moves which make $u \mathrm{OFF}$ and so we arrive at the required configuration $\mathbf{z}$.

CASE 2: Either $u$ or $v$ is not a corner vertex of $G$. There exist $w_{1} \in N_{G}(u) \backslash\{v\}$ and $w_{2} \in N_{G}(v) \backslash\{u, v\}$ such that $w_{1} w_{2} \notin E(G)$. After reverse-moves at $w_{2}, u$ and $w_{1}$ in that order, both $u$ and $v$ are $\mathrm{OFF}$ and hence the resulting configuration can be chosen to be $\mathbf{z}$.

Proof of Theorem 20. Pick any vertex $v$ from $L$. Part of $\mathcal{S}(G)$ can be drawn as in Fig. 11. Depending on the position of $v$ in $G$, some $w_{i}$ may be nonexistent. But, without loss of generality, we can always assume the existence of $w_{6}, w_{7}, w_{11}$. Denote by $N$ the 


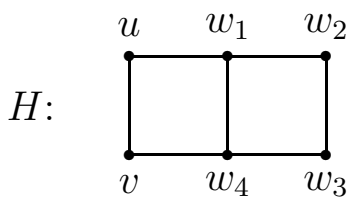

Figure 10: Both $u$ and $v$ are corner vertices.

\begin{tabular}{ll|l|l|l} 
& $w_{1}$ & $w_{2}$ & $w_{3}$ & $w_{4}$ \\
& $w_{5}$ & $w_{6}$ & $w_{7}$ & $w_{8}$ \\
& & & & \\
$w_{9}$ & $v$ & $w_{11}$ & $w_{12}$ \\
\hline & $w_{13}$ & $w_{14}$ & $w_{15}$ & $w_{16}$ \\
\hline & & &
\end{tabular}

Figure 11: Part of $\mathcal{S}(G)$ around $v, w_{6}, w_{7}, w_{11}$.

set $\left\{w_{1}, w_{2}, w_{5}, w_{6}, w_{7}, v, w_{11}, w_{12}, w_{15}, w_{16}\right\}$ and use the notation $N^{\prime}$ for $N \backslash\left\{w_{7}, v\right\}$. We depict the shadow graph of the induced subgraph $G[N]$ in Fig. 12.

By Lemma 34, there exists $\mathbf{z} \in \mathbb{F}_{2}^{V(G)}$ such that

$$
\mathbf{z} \underset{Q_{1}}{\stackrel{*}{\longrightarrow}} \mathbf{y}
$$

for some subset $Q_{1}$ of $V(G)$ and $\mathbf{z}(v)=0$. Moreover, Lemma 35 allows us to assume that

$$
\mathbf{z}\left(w_{6}\right)=0 \quad \text { when } \quad w_{6} \notin L .
$$

We then play the lit-only $\sigma$-game on $G$ with the initial configuration x. Since $m \geq 2$ and $n \geq 3$, we can find that $N_{G}\left(w_{6}\right) \neq N_{G}\left(w_{11}\right)$ and so Lemma 28 guarantees that there exist $Q_{2} \subseteq V(G)$ and $\overline{\mathbf{x}} \in \mathbb{F}_{2}^{V(G)}$ such that

$$
\mathbf{x} \underset{Q_{2}}{\stackrel{*}{\longrightarrow}} \overline{\mathbf{x}} \text { and } \overline{\mathbf{x}}\left(w_{6}\right) \neq \overline{\mathbf{x}}\left(w_{11}\right) .
$$

Due to the topological structure of the grid graph (see Fig. 11), we know that $G\left[V(G) \backslash N^{\prime}\right]$ has at most two components, say $S_{1}$ that contains $v$ and $S_{2}$ that contains $w_{7}$. Note that $G\left[V(G) \backslash N^{\prime}\right]$ is connected if and only if $S_{1}=S_{2}$. We claim that there exists $\bar{S} \subseteq N^{\prime}$ such that for $\mathbf{x}^{\prime}=\mathbf{z}+\sum_{w \in \bar{S}} \chi_{N_{G}(w)}$ we have

$$
\overline{\mathbf{x}} \underset{Q_{1} \triangle Q_{2} \triangle Q \triangle \bar{S}}{G} \mathbf{x}^{\prime} \text { and } \mathbf{x}^{\prime}\left(w_{6}\right) \neq \mathbf{x}^{\prime}\left(w_{11}\right) .
$$

Applying Lemma 29 for $(a, b, c, S)=\left(w_{6}, w_{11}, v, S_{1} \backslash\{v\}\right)$, we find that Eq. (8) holds for some $\bar{S} \subseteq V(G) \backslash S_{1}$. If $S_{1}=S_{2}$, then $V(G) \backslash S_{1}=N^{\prime}$ and so we are done. If $S_{1} \neq S_{2}$, we 


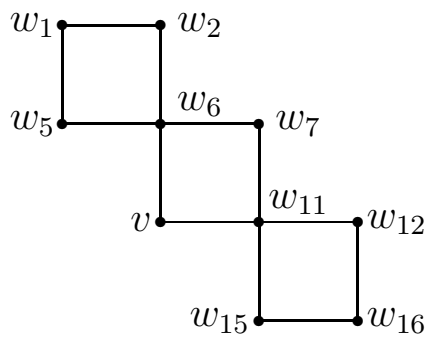

Figure 12: The graph $\mathcal{S}(G)[N]$.

can employ Lemma 29 once again with the choice of $(a, b, c, S)=\left(w_{6}, w_{11}, w_{7}, S_{2} \backslash\left\{w_{7}\right\}\right)$ and further reduce the range of $\bar{S}$ to make it inside $N^{\prime}$, thus proving the claim.

To proceed, we turn our attention to Fig. 12 and play the lit-only $\sigma$-game on $G$ with initial configuration $\mathbf{x}^{\prime}$ restricted to moves inside $N$. Since one of $w_{6}$ and $w_{11}$ is $\mathrm{ON}$, we can turn $v$ ON, and then, by a possible toggling at $v \in L$, make the states of $v$ and $w_{7}$ different. Applying Lemma 29 at this moment for $(a, b, c)=\left(v, w_{7}, w_{6}\right)$ and $\left(v, w_{7}, w_{11}\right)$, we know from Eqs. (7) and (8) that

$$
\mathbf{x} \underset{Q \triangle Q_{1} \triangle S^{\prime}}{\stackrel{*}{\longrightarrow}} \mathbf{x}^{\prime \prime}=\mathbf{z}+\sum_{w \in S^{\prime}} \chi_{N_{G}(w)}
$$

for some $S^{\prime} \subseteq\left\{v, w_{7}\right\}$ and $\mathbf{x}^{\prime \prime}(v) \neq \mathbf{x}^{\prime \prime}\left(w_{7}\right)$.

In view of Eqs. (5) and (9), to complete the proof we need to show that

$$
\mathbf{x}^{\prime \prime} \underset{S^{\prime}}{\stackrel{*}{\longrightarrow}} \mathbf{z}
$$

From $v \in N_{G}(v) \backslash N_{G}\left(w_{7}\right)$ we deduce that $\chi_{N_{G}(v)}$ and $\chi_{N_{G}\left(w_{7}\right)}$ are linearly independent and so it suffices to establish

$$
\mathbf{x}^{\prime \prime} \underset{S^{\prime \prime}}{\stackrel{*}{\longrightarrow}} \mathbf{z}
$$

for any $S^{\prime \prime} \subseteq\left\{v, w_{7}\right\}$ (which can only be $S^{\prime}$ ). We focus on the path $\left[v, w_{6}, w_{7}\right]$ and construct the required sequence of valid moves in all the possibilities.

CASE 1: $\left(\mathbf{x}^{\prime \prime}(v), \mathbf{x}^{\prime \prime}\left(w_{7}\right)\right)=(0,1)$.

Since $\mathbf{z}(v)=0$ and $v \in L$, we conclude that either $\mathbf{z}=\mathbf{x}^{\prime \prime}$ or $\mathbf{z}=\mathbf{x}^{\prime \prime}+\chi_{N_{G}\left(w_{7}\right)}$. As $\mathbf{x}^{\prime \prime}\left(w_{7}\right)=1$, a possible lit-only move at $w_{7}$ brings us from $\mathbf{x}^{\prime \prime}$ to $\mathbf{z}$.

CASE 2: $\left(\mathbf{x}^{\prime \prime}(v), \mathbf{x}^{\prime \prime}\left(w_{7}\right)\right)=(1,0)$. Since $\mathbf{z}(v)=0$, we have $S^{\prime}=\{v\}$ or $\left\{v, w_{7}\right\}$.

CASE 2.1: $S^{\prime}=\{v\}$. This is trivial as $\mathbf{x}^{\prime \prime}(v)=1$.

CASE 2.2: $S^{\prime}=\left\{v, w_{7}\right\}$. We now have $\mathbf{z}=\mathbf{x}^{\prime \prime}+\chi_{N_{G}(v)}+\chi_{N_{G}\left(w_{7}\right)}$ and hence $\mathbf{z}\left(w_{6}\right)=$ $\mathbf{x}^{\prime \prime}\left(w_{6}\right)$. Recall from Eq. (6) that if $w_{6} \notin L$ then we have $\mathbf{z}\left(w_{6}\right)=0$. Therefore, the following cases are exhaustive.

CASE 2.2.1: $w_{6} \notin L, \mathbf{x}^{\prime \prime}\left(w_{6}\right)=0$. It is not hard to check that the sequence of lit-only moves at $v, w_{6}, w_{7}, v, w_{6}, v$ transforms $\mathbf{x}^{\prime \prime}$ to $\mathbf{z}$.

CASE 2.2.2: $w_{6} \in L, \mathbf{x}^{\prime \prime}\left(w_{6}\right)=0$. The sequence of lit-only moves at $v, w_{6}, w_{7}, w_{6}$ does it. 
CASE 2.2.3: $w_{6} \in L, \mathbf{x}^{\prime \prime}\left(w_{6}\right)=1$. The required sequence of lit-only moves can be chosen to be $w_{6}, w_{7}, w_{6}, v$.

Lemma 36. Let $G$ be a graph with $\mathcal{S}(G)=G_{2,2}$ and $V(G) \cap E(G)=L \neq \emptyset$. For any $\mathbf{x} \in \mathbb{F}_{2}^{V(G)} \backslash\{\mathbf{0}\}$ and $\mathbf{y} \in \mathbb{F}_{2}^{V(G)} \backslash\left\{\chi_{L}\right\}$, if $\mathbf{x} \rightarrow_{G} \mathbf{y}$ then $\mathbf{x} \stackrel{*}{\rightarrow}_{G} \mathbf{y}$. In particular, $\mathcal{D}(G)=0$.

Proof. When $|L|=1,2,3$, the claim follows from an exhausted enumeration based on those phase spaces given in Appendix A (Figs. 14, 15, 16 and 17). For $|L|=4$, the result turns out to be a special case of Example 8.

Proof of Theorem 21. Theorem 20 together with Lemma 36 proves the result, as desired.

Proof of Theorem 22. This follows from the combination of Theorem 21 and Sutner's Theorem (Example 7).

\section{Paths}

Lemma 37. Let $n \geq 2, P$ be a pseudo-path such that $\mathcal{S}(P)=\left[v_{1}, v_{2}, \ldots, v_{n}\right]$. If $P$ is singular, then $P-v_{n}$ is nonsingular.

Proof. Let $t_{i}$ be the determinant of the adjacency matrix of $P\left[\left\{v_{1}, \ldots, v_{i}\right\}\right]$ for $1 \leq i \leq n$ and let $t_{0}=1$. It is clear that

$$
t_{i}=\ell_{i} t_{i-1}+t_{i-2}, i=2, \ldots, n,
$$

where $\ell_{i}=1$ if $P$ has a loop at $v_{i}$ and $\ell_{i}=0$ else. It suffices to show that $t_{i}$ is 0 will imply $t_{i-1}$ is 1 for $i=1, \ldots, n$. When $i=1$, the result is clear. If the result is not true, there is a smallest $i \geq 2$ such that $t_{i}=t_{i-1}=0$. By the assumption on $i$, we know that $t_{i-2}=1$. But it follows from Eq. (10) that $t_{i-2}=0$, a contradiction.

Proof of Theorem 23. Suppose that $\mathcal{S}(P)=\left[v_{1}, v_{2}, \ldots, v_{n}\right]$. We shall prove the theorem by induction on $n$. The theorem is trivially true for $n=1$. Let us assume $n \geq 2$ and carry out the inductive step. Without loss of generality, suppose that $\mathbf{x} \neq \mathbf{0}$ and $P-v_{n}$ has a loop. By applying lit-only togglings on $\mathbf{x}$ to turn $v_{n} \mathrm{ON}$ and then making a possible move at $v_{n}$, we find a $\mathbf{y} \in \mathbb{F}_{2}^{V(G)}$ such that $\mathbf{x} \stackrel{*}{\rightarrow} \mathbf{y}$ and

$$
\mathbf{y}=\sum_{v \in S} \chi_{N_{G}(v)}
$$

for some $S \subseteq \Pi=\left\{v_{1}, \ldots, v_{n-1}\right\}$.

CASE 1: $\mathbf{y}=\mathbf{0}$. The result is trivially true.

CASE 2: $\mathbf{y} \neq \mathbf{0}, P-v_{n}$ is nonsingular. By the inductive hypotheses, $\mathbf{y} \underset{S^{\prime}}{\stackrel{*}{\longrightarrow}} t \chi_{v_{n}}$ for some $S^{\prime} \subseteq \Pi$ and $t \in \mathbb{F}_{2}$. Compared with Eq. (11), we obtain

$$
\sum_{v \in S} \chi_{N_{G}(v) \cap \Pi}=\sum_{v \in S^{\prime}} \chi_{N_{G}(v) \cap \Pi}
$$

THE ELECTRONiC JouRnal of COMBinatorics 18 (2011), \#P214 
Henceforth, we derive $S=S^{\prime}$ from the fact that $P-v_{n}$ is nonsingular. This in turn gives $t=0$ and $\mathbf{x} \stackrel{*}{\rightarrow} \mathbf{y} \underset{S}{\stackrel{*}{\rightarrow}} \mathbf{0}$, as desired.

CASE 3: $\mathbf{y} \neq \mathbf{0}, P-v_{n}$ is singular. Since $P-v_{n}$ has at least one loop, we know that $n \geq 3$. By Lemma 37 , both $P-v_{n}-v_{1}$ and $P-v_{n}-v_{n-1}$ are nonsingular.

SubCASE 3.1: $\mathbf{y}_{\Pi} \neq \mathbf{0}$. Clearly, we can pick a $v \in\left\{v_{1}, v_{n-1}\right\}$ such that $P-v_{n}-v$ has a loop. By a sequence of lit-only togglings on $P-v_{n}$, we can make $v$ ON and then a further possible pushing at $v$ produces a configuration $\mathbf{z}$ such that $\mathbf{y} \stackrel{*}{\rightarrow} \mathbf{z}$ and $\mathbf{z}$ can be turned all-OFF by regular togglings on $P-v_{n}-v$. By the inductive hypotheses, there is a sequence of lit-only togglings on $P-v_{n}-v$ which brings $\mathbf{z}$ to $\mathbf{w}$ where $\mathbf{w}$ can only take nonzero value on either $v$ or $v_{n}$. Since $\mathbf{w}$ can be turned all-OFF by regular togglings on $P-v_{n}-v$ and $P-v_{n}-v$ is nonsingular, $\mathbf{w}(v)=\mathbf{w}\left(v_{n}\right)=0$ and hence $\mathbf{w}=\mathbf{0}$.

SubCASE 3.2: $\mathbf{y}_{\Pi}=\mathbf{0}$, namely $\mathbf{y}=\chi_{v_{n}}$. Let $k$ be the largest integer such that $v_{k}$ has a loop.

SubCase 3.2.1: $v_{n} \notin E(P)$, or equivalently, $k<n$. By executing the sequential togglings at $v_{n}, v_{n-1}, \ldots, v_{k}$, we see that

$$
\mathbf{y} \stackrel{*}{\rightarrow} \chi_{v_{k-1}} .
$$

If $k=1$, we are finished. Otherwise, after the additional sequential togglings at $v_{k-1}$, $v_{k}, \ldots, v_{n}$, we see that $\mathbf{y} \stackrel{*}{\rightarrow} \mathbf{z}$ where $\mathbf{z}\left(v_{k}\right)=1$ and $\mathbf{z}$ can be turned all-OFF by regular togglings on $P-v_{n}$ (as we toggle $v_{n}$ twice in the process of going from $\mathbf{y}$ to $\mathbf{z}$ ). We are now reduced to Subcase 3.1.

SubCASE 3.2.2: $v_{n} \in E(P)$, or equivalently, $k=n$. By toggling $v_{n}, v_{n-1}$ and $v_{n}$ consecutively, we reach a configuration $\mathbf{u}$ with $\mathbf{u}\left(v_{n-2}\right)=1$. Observe that $\mathbf{u}$ can still be turned all-OFF by regular moves inside $P-v_{n}$. This means that we return to Subcase 3.1 with the new $\mathbf{y}$ being $\mathbf{u}$.

Lemma 38. Let $P$ be obtained from a path by attaching a loop at one of its endpoints. Then $P$ is nonsingular.

Proof. For both the case of $|V(P)|$ being odd and $|V(P)|$ being even, we can check that the Laplace expansion of the determinant of the adjacency matrix of $P$ has only one nonzero term and hence the result follows. The result can also be seen by verifying that there is no nonempty set $S \subseteq V(P)$ such that each vertex of $P$ is adjacent to an even number of vertices in $S$.

Proof of Theorem 24. Let $v$ be $v_{1}$ or $v_{n}$. Let us show that $\mathbf{x} \stackrel{*}{\rightarrow} \chi_{v}$. Since $\mathbf{x}$ cannot be reduced to $\mathbf{0}$ by valid togglings, we know from Theorem 23 that $P$ is singular. Lemma 38 now tells us that $v$ cannot be the only loop of $P$ and so $P-v$ is not loopless. Meanwhile, we obtain from Lemma 37 that $P-v$ is nonsingular. To conclude the proof, we first choose a $\mathbf{y}$ such that $\mathbf{x} \stackrel{*}{\rightarrow} \mathbf{y}$ and $\mathbf{y}(v)=1$, which is possible as $\mathbf{x}$ cannot be $\mathbf{0}$, and then appeal to Theorem 23 for $\mathbf{y}$ restricted on $P-v$. 


\section{Grid graphs}

Lemma 39. Let $G=G_{m, n}$ and $C=\left\{v_{1, j}: 1 \leq j \leq n-1\right\} \cup\left\{v_{i, 1}: 1 \leq i \leq m-1\right\}$. For any $\mathbf{x} \in \mathbb{F}_{2}^{V(G)}$, there exists $\mathbf{y} \in \mathbb{F}_{2}^{V(G)}$ such that $\mathbf{y}_{C}=\mathbf{0}, L(\mathbf{y}) \leq L(\mathbf{x})$ and $\mathbf{x} \rightarrow_{G} \mathbf{y}$.

Proof. If $\mathbf{x}\left(v_{k, 1}\right)=1$ and $k \leq m-1$, then

$$
\mathbf{z}=\mathbf{x}+\sum_{i=1}^{\min \{n, m-k\}} \chi_{N_{G}\left(v_{k+i, i}\right)}
$$

satisfies $L(\mathbf{z}) \in\{L(\mathbf{x}), L(\mathbf{x})-1, L(\mathbf{x})-2\}, \mathbf{z}_{C \backslash\left\{v_{k, 1}\right\}}=\mathbf{x}_{C \backslash\left\{v_{k, 1}\right\}}$ and $\mathbf{z}\left(v_{k, 1}\right)=0$. Similarly, if $\mathbf{x}\left(v_{1, k}\right)=1$ and $k \leq n-1$, then

$$
\mathbf{z}=\mathbf{x}+\sum_{i=1}^{\min \{m, n-k\}} \chi_{N_{G}\left(v_{i, k+i}\right)}
$$

satisfies $L(\mathbf{z}) \in\{L(\mathbf{x}), L(\mathbf{x})-1, L(\mathbf{x})-2\}, \mathbf{z}_{C \backslash\left\{v_{1, k}\right\}}=\mathbf{x}_{C \backslash\left\{v_{1, k}\right\}}$ and $\mathbf{z}\left(v_{1, k}\right)=0$. It then follows that the required $\mathbf{y}$ can be taken to be

$$
\mathbf{y}=\mathbf{x}+\sum_{\substack{v_{k, 1} \in \operatorname{supp}(\mathbf{x}) \\ k \leq m-1}} \sum_{i=1}^{\min \{n, m-k\}} \chi_{N_{G}\left(v_{k+i, i}\right)}+\sum_{\substack{v_{1, k} \in \operatorname{supp}(\mathbf{x}) \\ k \leq n-1}} \sum_{i=1}^{\min \{m, n-k\}} \chi_{N_{G}\left(v_{i, k+i}\right)}
$$

finishing the proof.

Proof of Theorem 25. Write $G$ for $G_{m, n}$. If $\min (m, n) \leq 2$, then it follows from Theorem 18 (Theorem 19) that $M L^{*}(G) \leq 2$ and so the result is clear. If $\mathbf{x}=\mathbf{0}$, then $M L_{G}^{*}(\mathbf{x})=$ $M L_{G}(\mathbf{x})=0$.

We now assume that $\min (m, n) \geq 3, \mathbf{x} \in \mathbb{F}_{2}^{V(G)} \backslash\{\mathbf{0}\}$, and try to show that $M L_{G}^{*}(\mathbf{x})-M L_{G}(\mathbf{x}) \leq 2$. Just for convenience, we also refer to $v_{3,1}, v_{1,3}, v_{2,1}, v_{1,2}, v_{1,1}$ as $u_{1}, u_{2}, u_{3}, u_{4}, u_{5}$, respectively; see Fig. 13 . Denote by $S_{k}$ the set $\left\{u_{1}, u_{2}, \ldots, u_{5}\right\} \backslash$ $\left\{u_{1}, u_{2}, \ldots, u_{k-1}\right\}$ for $1 \leq k \leq 6$. Note that $G\left[S_{k}\right]$ is connected for any $k$.

By Lemma 28, we can assume that $u_{3}$ and $u_{4}$ have different states in $\mathbf{x}$. Take $\mathbf{y}$ such that $\mathbf{x} \rightarrow_{G} \mathbf{y}$ and $L(\mathbf{y})=M L_{G}(\mathbf{x})$. By Lemma 39, we may suppose that $\mathbf{y}\left(v_{i, 1}\right)=0$ for all $i \in\{1, \ldots, m-1\}$ and $\mathbf{y}\left(v_{1, j}\right)=0$ for all $j \in\{1, \ldots, n-1\}$.

Setting $a=u_{3}, b=u_{4}, c=v_{2,2}$ and $S=V(G) \backslash\left(S_{1} \cup\left\{v_{2,2}\right\}\right)$, we deduce from Lemma 29 that there exists $R_{1} \subseteq S_{1}$ such that $\mathbf{x} \stackrel{*}{\rightarrow}_{G} \mathbf{y}+\sum_{v \in R_{1}} \chi_{N_{G}(v)}=\mathbf{z}_{1}$ and $\mathbf{z}_{1}\left(u_{3}\right) \neq \mathbf{z}_{1}\left(u_{4}\right)$.

Since one of $u_{3}$ and $u_{4}$ must be ON in $\mathbf{z}_{1}$ and $G\left[S_{1}\right]$ is connected, we can apply our basic strategy [45, Sec. 3] to yield $\mathbf{x} \stackrel{*}{\rightarrow}_{G} \mathbf{z}_{1} \stackrel{*}{\rightarrow}_{G} \mathbf{y}+\sum_{v \in R_{2}} \chi_{N_{G}(v)}=\mathbf{z}_{2}$ for some $R_{2} \subseteq S_{2}$, regardless of the value of $\mathbf{z}_{1}\left(u_{1}\right)$ and the position of $u_{1}$ relative to $R_{1}$.

Going this way, let us find inductively for $k=3,4,5,6$ and $R_{k} \subseteq S_{k}$ such that

$$
\mathbf{x} \stackrel{*}{\rightarrow}_{G} \mathbf{y}+\sum_{v \in R_{k}} \chi_{N_{G}(v)}=\mathbf{z}_{k} .
$$




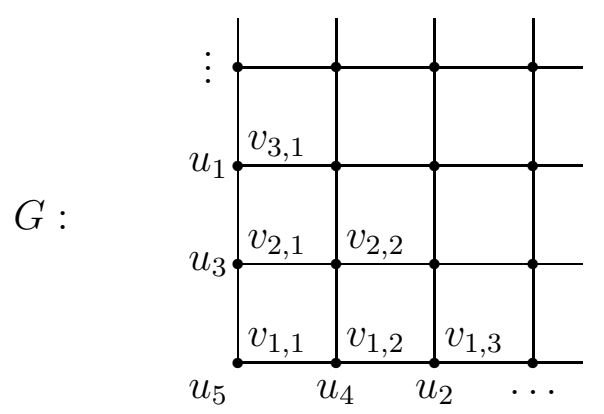

Figure 13: Locations of $u_{1}, \ldots, u_{5}$ in the grid graph $G$.

If this can be done till $k=6$, we have $\mathbf{x} \stackrel{*}{\rightarrow}_{G} \mathbf{z}_{6}=\mathbf{y}$ and so $M L_{G}^{*}(\mathbf{x})=M L_{G}(\mathbf{x})$ follows. Otherwise, there is the smallest $k \in\{3,4,5,6\}$ at which we get stuck. This implies that for this $k \in\{3,4,5,6\}$ we can find an $R_{k-1} \subseteq S_{k-1}$ such that $u_{k-1} \in R_{k-1}$,

$$
\mathbf{x} \stackrel{*}{\rightarrow}_{G} \mathbf{y}+\sum_{v \in R_{k-1}} \chi_{N_{G}(v)}=\mathbf{z}_{k-1}
$$

and

$$
\left|\operatorname{supp}\left(\mathbf{z}_{k-1}\right) \cap S_{k-1}\right|=0 .
$$

If $k=6, L\left(\mathbf{z}_{5}\right)-L(\mathbf{y}) \leq\left|\operatorname{supp}\left(\chi_{N_{G}\left(u_{5}\right)}\right)\right|=\left|\left\{u_{3}, u_{4}\right\}\right|=2$. If $k=3$ and $\mathbf{y}\left(u_{2}\right)=0$ or if $k=5$, since the path $G\left[S_{2}\right]$ and $G\left[S_{4}\right]$ are nonsingular, we have $\mathbf{z}_{k-1}=\mathbf{y}$. If $k=4$, then $R=\left\{u_{3}, u_{4}\right\}$ or $R=\emptyset$, so $L\left(\mathbf{z}_{3}\right)-L(\mathbf{y}) \leq\left|\operatorname{supp}\left(\chi_{N_{G}\left(u_{3}\right)}+\chi_{N_{G}\left(u_{4}\right)}\right)\right|=\left|\left\{u_{1}, u_{2}\right\}\right|=2$. If $k=3$ and $\mathbf{y}\left(u_{2}\right)=1$, which can happen only if $n=3$, we have $R=\left\{u_{3}, u_{4}\right\}$ and hence $L\left(\mathbf{z}_{2}\right)-L(\mathbf{y}) \leq\left|\operatorname{supp}\left(\chi_{N_{G}\left(u_{3}\right)}+\chi_{N_{G}\left(u_{4}\right)}\right) \backslash\left\{u_{2}\right\}\right|=\left|\left\{u_{1}\right\}\right|=1$. In conclusion, $M L_{G}^{*}(\mathbf{x})-M L_{G}(\mathbf{x}) \leq 2$, and hence the theorem, is established.

Our proof of Theorem 25 only relies on the local structure at the corner of the grid graphs. The work of Florence and Meunier [11] is the very rare one which contains results and conjectures for the $\sigma$-game on general $k$-dimensional grid graphs. It might be interesting to pursue the counterpart of Theorem 25 for general grid graphs by investigating how good the higher-dimensional corner could allow us to play the lit-only $\sigma$-game equipped with information about the best way to play the $\sigma$-game.

Acknowledgements This work was supported by the Fundamental Research Funds for the Central Universities of China, the NNSFC (No. 10871128), the STCSM (No. 09XD1402500) and the Chinese Ministry of Education (No. 108056). Y. Wu thanks the hospitality of West Virginia University during his visit there in the summer of 2009 as well as the support of Banff International Research Station during the Banff workshop "Linear Algebraic Techniques in Combinatorics/Graph Theory", Jan. 30 - Feb. 4, 2011. We thank the anonymous referee for a very careful reading which helps to improve our paper. 


\section{References}

[1] B. Bagchi, N.S.N. Sastry, Even order inversive planes, generalized quadrangles and codes, Geometriae Dedicata 22 (1987), 137-147.

[2] R. Barua, S. Ramakrishnan, $\sigma$-game, $\sigma^{+}$-game and two-dimensional additive cellular automata, Theoretical Computer Science 154 (1996), 349-366.

[3] William Y.C. Chen, Nancy S.S. Gu, Loop deletion for the lamp lighting problem, preprint. Available online at: http://www.billchen.org/preprint/lamp/lamp.pdf.

[4] G.J. Chang, M-K. Chuah, Vogan diagrams and the classification of real simple Lie algebras, Proceedings of the 4th International Congress of Chinese Mathematicians, (L. Ji, K. Liu, L. Yang, S-T. Yau, Eds.), Vol. I, Higher Education Press, pp. 385-398, 2008. Available online at: http://intlpress.com/_pages/special/iccm2007/pdf/03_03_01_ChangGerard.pdf.

[5] M-K. Chuah, C-C. Hu, Equivalence classes of Vogan diagrams, Journal of Algebra 279 (2004), 22-37.

[6] M-K. Chuah, C-C. Hu, Extended Vogan diagrams, Journal of Algebra 301 (2006), 112-147.

[7] M-K. Chuah, C-C. Hu, A quick proof on the equivalence of extended Vogan diagrams, Journal of Algebra 313 (2007), 824-827.

[8] D. Craft, Z. Miller, D. Pritikin, A solitaire game played on 2-colored graphs, Discrete Mathematics 309 (2009), 188-201.

[9] Y. Dodis, P. Winkler, Universal configurations in light-flipping games, in: Proc. of 12th Annunal ACM/SIAM Symposium on Discrete Algorithms, January 2001, pp. 926-927.

[10] H. Eriksson, K. Eriksson, J. Sjöstrand, Note on the lamp lighting problem, Special issue in honor of Dominique Foata's 65th birthday (Philadelphia, PA, 2000), Advances in Applied Mathematics 27 (2001), 357-366.

[11] M. Florence, F. Meunier, Completely symmetric configurations for $\sigma$-games on grid graphs, Journal of Algebraic Combinatorics 31 (2010), 533-545.

[12] A.S. Fraenkel, Error-correcting codes derived from combinatorial games, in: Games of No Chance, Proc. MSRI Workshop on Combinatorial Games, July, 1994, Berkeley, CA (R. J. Nowakowski, ed.), MSRI Publ. Vol. 29, Cambridge University Press, Cambridge, pp. 417-431, 1996.

[13] A.S. Fraenkel, Virus versus mankind, Lecture Notes in Computer Science 2063 (2001), 204-213.

[14] A.S. Fraenkel, Mathematical chats between two physicists, in Puzzlers' Tribute: A Feast for the Mind, honoring Martin Gardner (D. Wolfe and T. Rodgers, eds.), A K Peters, 2002, pp. 315-325.

[15] A.S. Fraenkel, Two-player games on cellular automata, More Games of No Chance, Proc. MSRI Workshop on Combinatorial Games, July 10 - 28, 2000, Berkeley, CA (R.J. Nowakowski, ed.), MSRI Publ. Vol. 42, Cambridge University Press, Cambridge, pp. 279 306, 2002.

[16] A.S. Fraenkel, Combinatorial games, The Electronical Journal of Combinatorics DS2 (2009), $88 \mathrm{pp}$. 
[17] A.S. Fraenkel, O. Rahat, Complexity of error-correcting codes derived from combinatorial games, Lecture Notes in Computer Science 2883 (2003), 201-212.

[18] S.V. Gervacio, H. Maehara, A note on lights-out-puzzle: Parity-state graphs, Graphs and Combinatorics 27 (2011), 109-119.

[19] A. Giffen, D.B. Parker, On generalizing the Lights Out game and a generalization of parity domination, to appear in: Congressus Numerantium. Available online at: http://faculty . gvsu.edu/parkerda/profstuff/papers/hyperlogpd.pdf.

[20] J.L. Goldwasser, W.F. Klostermeyer, Maximization versions of "lights out" games in grids and graphs, Congressus Numerantium 126 (1997), 99-111.

[21] J.L. Goldwasser, W.F. Klostermeyer, G. Trapp, Characterizing switch-setting problems, Linear and Multilinear Algebra 43 (1997), 121-135.

[22] J. Goldwasser, W. Klostermeyer, H. Ware, Fibonacci polynomials and parity domination in grid graphs, Graphs and Combinatorics 18 (2002), 271-283.

[23] J.L. Goldwasser, W.F. Klostermeyer, Parity dominating sets in grid graphs, Congressus Numerantium 172 (2005), 79-96.

[24] J.L. Goldwasser, W.F. Klostermeyer, Odd and even dominating sets with open neighborhoods, Ars Combinatoria 83 (2007), 229-247.

[25] J.L. Goldwasser, W.F. Klostermeyer, Maximum orbit weights in the $\sigma$-game and lit-only $\sigma$-game on grids and graphs, Graphs and Combinatorics 25 (2009), 309-326.

[26] J. Goldwasser, X. Wang, Y. Wu, Does the lit-only restriction make any difference for the $\sigma$-game and $\sigma^{+}$-game? European Journal of Combinatorics 30 (2009), 774-787.

[27] J. Goldwasser, X. Wang, Y. Wu, Several kinds of reachability relationships for the phase space of the lit-only $\sigma$-game, in preparation.

[28] S. Gravier, M. Mhalla, E. Tannier, On a modular domination game, Theoretical Computer Science 306 (2003), 291-303.

[29] H-W. Huang, C-W. Weng, Combinatorial representations of Coxeter groups over a field of two elements, arXiv:0804.2150v2, 2010.

[30] H-W. Huang, C-W. Weng, The edge-flipping group of a graph, European Journal of Combinatorics 31 (2010), 932-942.

[31] H-W. Huang, C-W. Weng, The flipping puzzle on a graph, European Journal of Combinatorics 31 (2010), 1567-1578.

[32] H-W. Huang, Lit-only sigma-game on some trees, arXiv:1010.5846v1, 2010.

[33] G. Istrate, On the dynamics of social balance on general networks (with an application to XOR-SAT), Fundamenta Informaticae 91 (2009), 341-356.

[34] G. Istrate, Reachability and recurrence in a modular generalization of annihilating random walks (and lights-out games) on hypergraphs, arXiv:1102.1440v1, 2011.

[35] A. Lindenmayer, Mathematical models for cellular interactions in development, Journal of Theoretical Biology 18 (1968), 280-299.

[36] T. Muetze, Generalized switch-setting problems, Discrete Mathematics 307 (2007), 27552770 . 
[37] M. Nath, B.K. Sarma, On the null-spaces of acyclic and unicyclic singular graphs, Linear Algebra and its Applications 427 (2007), 42-54.

[38] P.M. Nylen, Minimum-rank matrices with prescribed graph, Linear Algebra and its Applications 248 (1996), 303-316.

[39] M. Reeder, Level-two structure of simply-laced Coxeter groups, Journal of Algebra 285 (2005), 29-57.

[40] J. Scherphuis, The Mathematics of Lights Out, http://www.jaapsch.net/puzzles/ lomath.htm.

[41] K. Sutner, Linear cellular automata and the Garden of Eden, The Mathemaitcal Intelligencer 11 (1989), 49-53.

[42] K. Sutner, The $\sigma$-game and cellular automata, American Mathematical Monthly 97 (1990), $24-34$.

[43] K. Sutner, $\sigma$-Automata and Chebyshev-polynomials, Theoretical Computer Science 230 (2000), 49-73.

[44] X. Wang, Y. Wu, Minimum light number of lit-only $\sigma$-game on a tree, Theoretical Computer Science 381 (2007), 292-300.

[45] X. Wang, Y. Wu, Lit-only $\sigma$-game on pseudo-trees, Discrete Applied Mathematics 158 (2010), 1945-1952.

[46] H-J. Wu, A study on equivalence classes of painted graphs, Master thesis, National Taiwan University, 2006.

[47] Y. Wu, Even poset and a parity result for binary linear code, Linear Algebra and its Applications 418 (2006), 591-594.

[48] Y. Wu, Lit-only sigma game on a line graph, European Journal of Combinatorics 30 (2009), $84-95$.

[49] M. Zaidenberg, Periodic harmonic functions on lattices and points count in positive characteristic, Central European Journal of Mathematics 7 (2009), 365-381.

\section{A Phase spaces of some pseudo-cycles}

We depict below the phase spaces of the lit-only $\sigma$-game on 4-cycles with one, two, or three loops attached. In each case, we display both the graph and the phase space of the lit-only $\sigma$-game on that graph. 

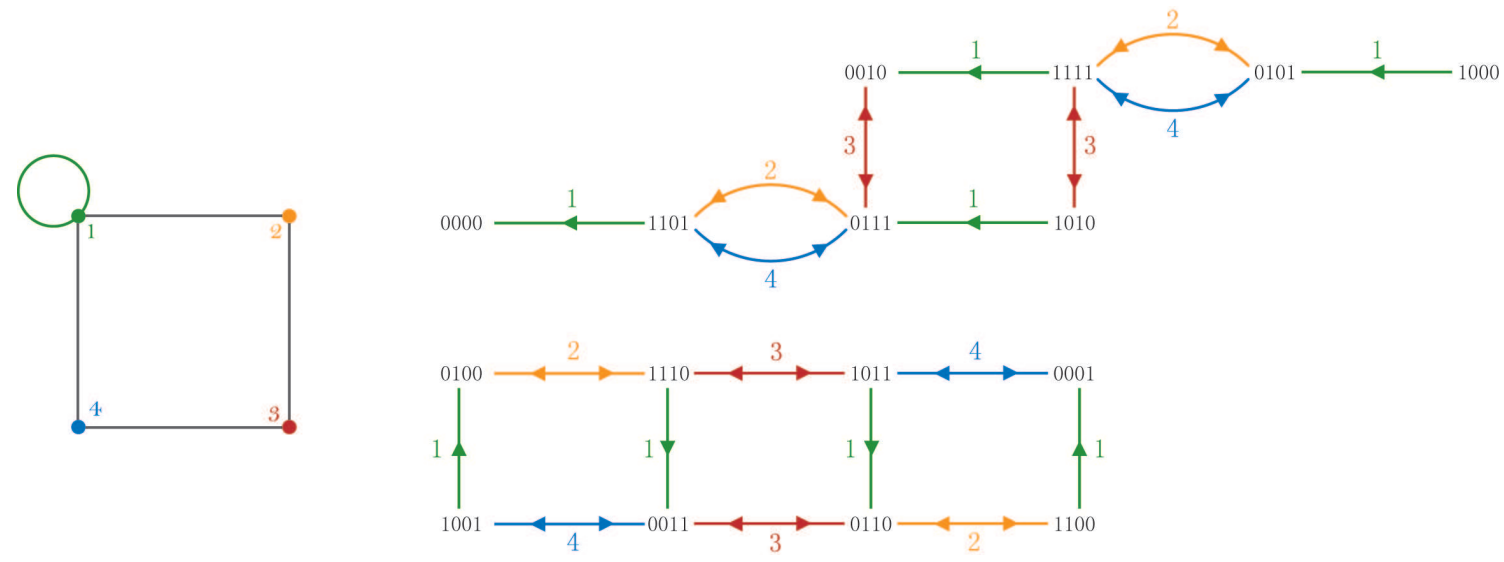

Figure 14: A 4-cycle with one loop and its phase space.
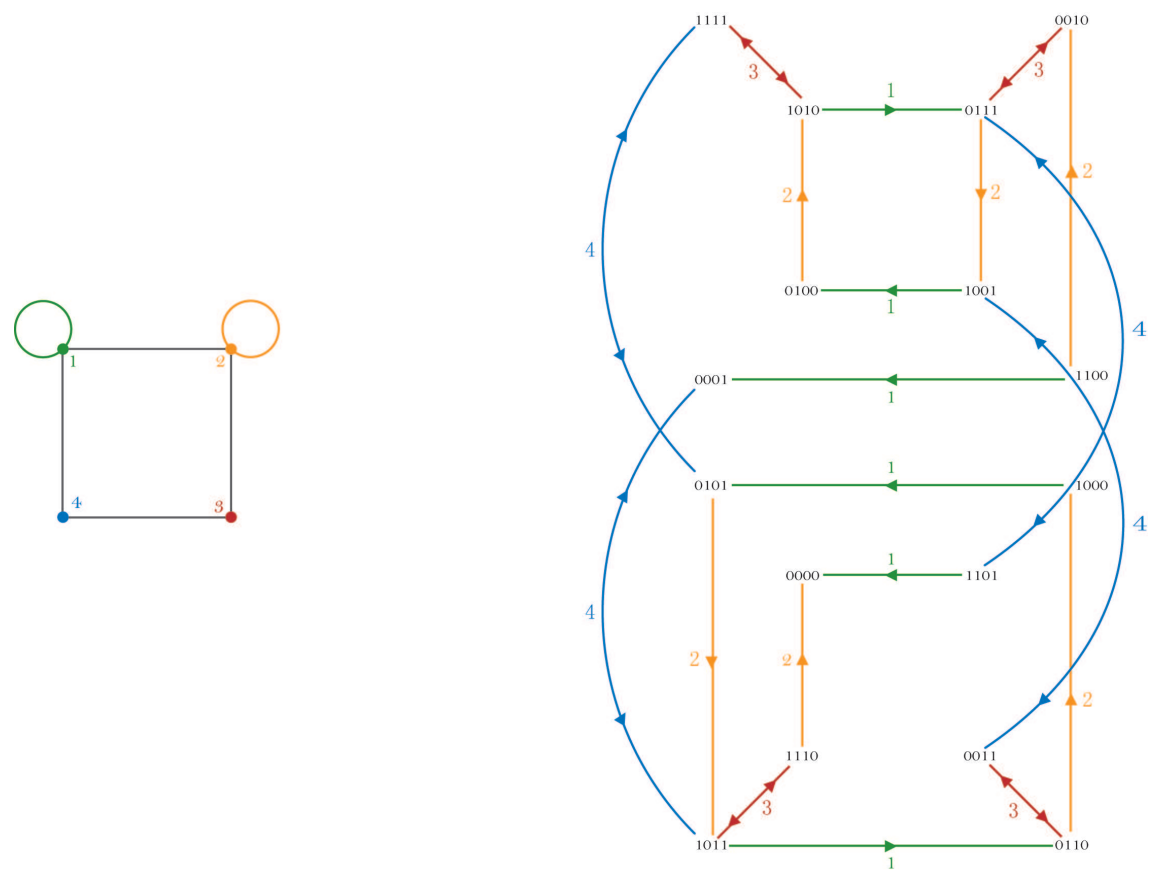

Figure 15: A 4-cycle with two adjacent loops and its phase space. 

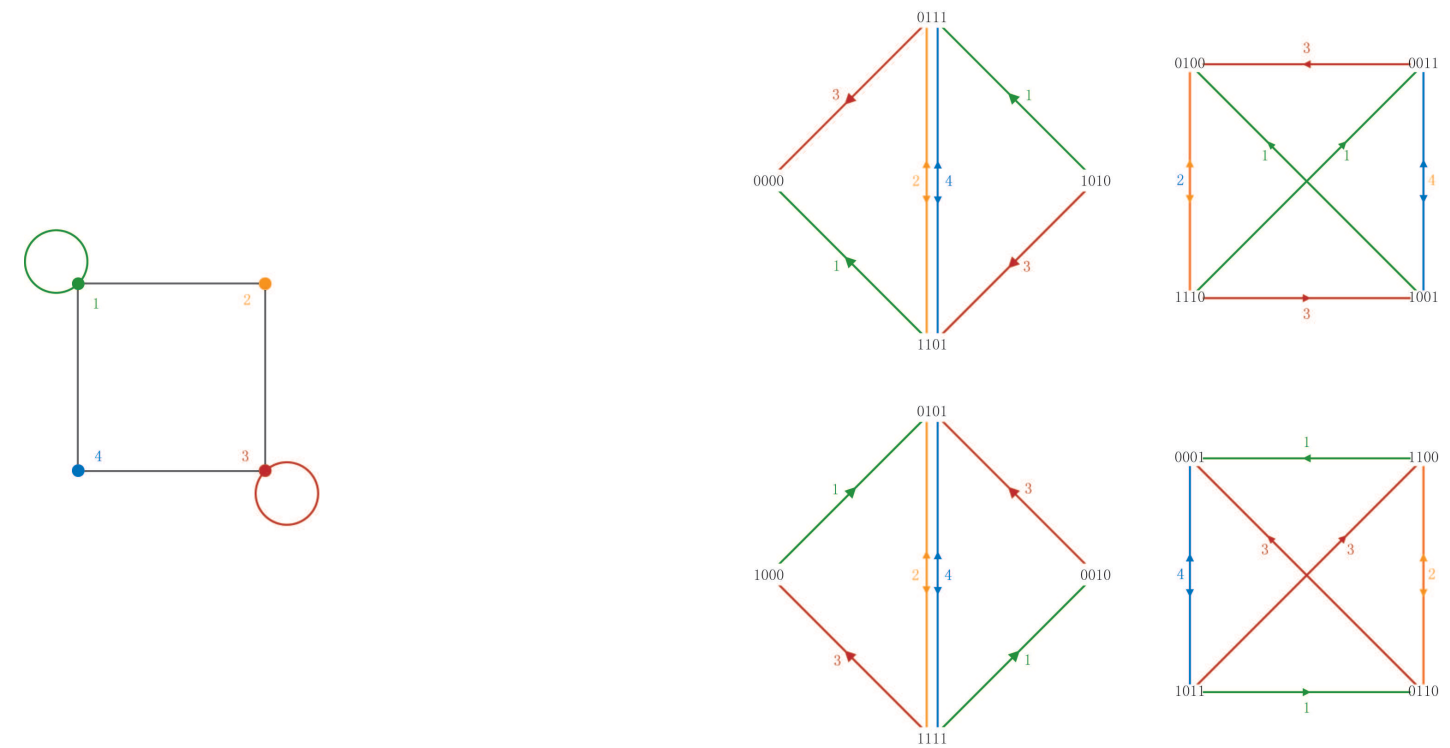

Figure 16: A 4-cycle with two nonadjacent loops and its phase space.
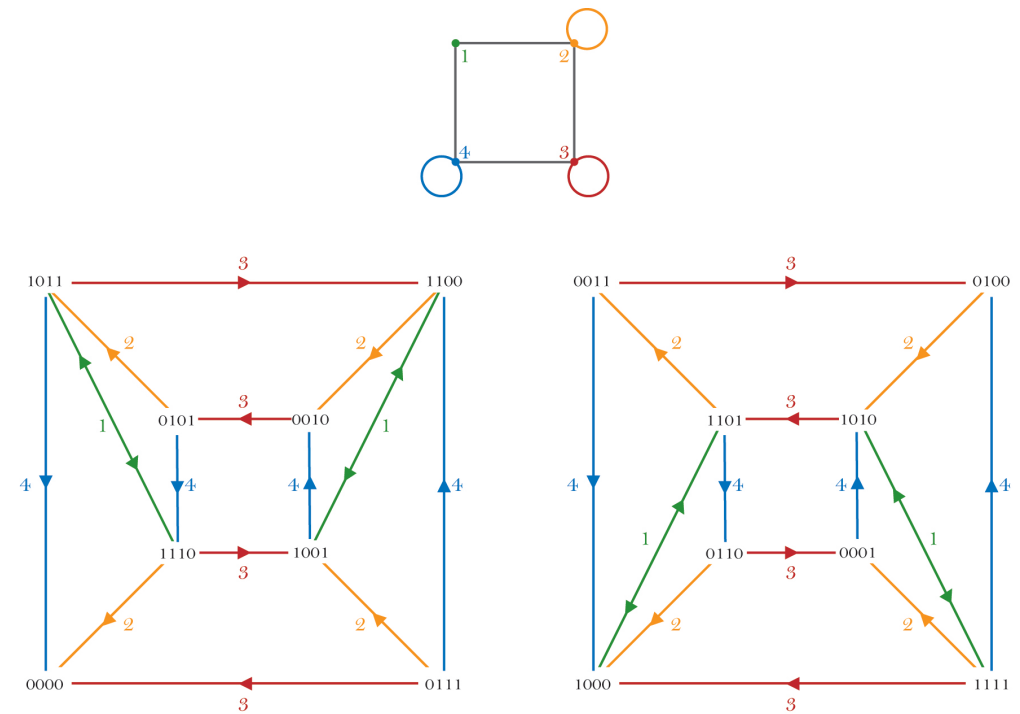

Figure 17: A 4-cycle with three loops and its phase space. 